\title{
Nitrous Oxide Emissions from Smallholders' Cropping Systems in Sub-Saharan Africa
}

\author{
Shaankua E. Lemarpe $\mathbb{D}^{1},{ }^{1}$ Collins M. Musafiri $\mathbb{D}^{1,},{ }^{1,2}$ Joseph M. Macharia $\mathbb{D}^{3}{ }^{3}$ \\ Milka N. Kiboi $\mathbb{D},{ }^{2}$ Onesmus K. Ng'etich, ${ }^{1}$ Chris A. Shisanya $\mathbb{D}^{3},{ }^{3}$ Jeremiah Okeyo $\mathbb{D},{ }^{1}$ \\ Elizabeth A. Okwuosa $\mathbb{D D}^{4}$ and Felix K. Ngetich $\mathbb{D D}^{2,5}$ \\ ${ }^{1}$ Department of Water and Agricultural Resource Management, University of Embu, P.O. Box 6-60100, Embu, Kenya \\ ${ }^{2}$ Cortile Scientific Co. Ltd., P.O. BOX 34991-00100, Nairobi, Kenya \\ ${ }^{3}$ Kenyatta University, Department of Geography, P.O. Box 43844-00100, Nairobi, Kenya \\ ${ }^{4}$ Kenya Agricultural and Livestock Research Organization (KALRO), Muguga, P.O. BOX 30148-00100, Nairobi, Kenya \\ ${ }^{5}$ Department of Plant, Animal and Food Sciences (PAFS), \\ Jaramogi Oginga Odinga University of Science and Technology (JOOUST), P.O. Box 210-40601, Bondo, Kenya
}

Correspondence should be addressed to Felix K. Ngetich; felixngetich@gmail.com

Received 23 April 2021; Accepted 16 October 2021; Published 1 November 2021

Academic Editor: Suhaib Bandh

Copyright (C) 2021 Shaankua E. Lemarpe et al. This is an open access article distributed under the Creative Commons Attribution License, which permits unrestricted use, distribution, and reproduction in any medium, provided the original work is properly cited.

\begin{abstract}
Increased concentration of atmospheric nitrous oxide $\left(\mathrm{N}_{2} \mathrm{O}\right)$, a potent greenhouse gas $(\mathrm{GHG})$, is of great concern due to its impact on ozone layer depletion leading to climate change. Ozone layer depletion allows penetration of ultraviolet radiations, which are hazardous to human health. Climate change culminates in reduced food productivity. Limited empirical studies have been conducted in Sub-Saharan Africa (SSA) to quantify and understand the dynamics of soil $\mathrm{N}_{2} \mathrm{O}$ fluxes from smallholder cropping systems. The available literature on soil $\mathrm{N}_{2} \mathrm{O}$ fluxes in SSA is limited; hence, there is a pressing need to consolidate it to ease mitigation targeting and policy formulation initiatives. We reviewed the state of $\mathrm{N}_{2} \mathrm{O}$ emissions from selected cropping systems, drivers that significantly influence $\mathrm{N}_{2} \mathrm{O}$ emissions, and probable soil $\mathrm{N}_{2} \mathrm{O}$ emissions mitigation options from 30 studies in SSA cropping systems have been elucidated here. The review outcome indicates that coffee, tea, maize, and vegetables emit $\mathrm{N}_{2} \mathrm{O}$ ranging from 1 to $1.9,0.4$ to $3.9,0.1$ to 4.26 , and 48 to $113.4 \mathrm{~kg} \mathrm{~N}_{2} \mathrm{O}-\mathrm{N} \mathrm{ha}^{-1} \mathrm{yr}^{-1}$, respectively. The yield-scaled and $\mathrm{N}_{2} \mathrm{O}$ emissions factors ranged between 0.08 and $67 \mathrm{~g} \mathrm{~N}_{2} \mathrm{O}-\mathrm{N} \mathrm{kg}^{-1}$ and 0.01 and $4.1 \%$, respectively, across cropping systems. Soil characteristics, farm management practices, and climatic and environmental conditions were significant drivers influencing $\mathrm{N}_{2} \mathrm{O}$ emissions across SSA cropping systems. We found that site-specific soil $\mathrm{N}_{2} \mathrm{O}$ emissions mitigation measures are required due to high variations in $\mathrm{N}_{2} \mathrm{O}$ drivers across SSA. We conclude that appropriate fertilizer and organic input management combined with improved soil management practices are potential approaches in $\mathrm{N}_{2} \mathrm{O}$ emissions mitigation in SSA. We recommend that (i) while formulating soil $\mathrm{N}_{2} \mathrm{O}$ emissions mitigation approaches, in SSA, policymakers should consider site-specific targeting approaches, and (ii) more empirical studies need to be conducted in diverse agroecological zones of SSA to qualify various mitigation options on $\mathrm{N}_{2} \mathrm{O}$ emissions, yield-scaled $\mathrm{N}_{2} \mathrm{O}$ emissions, and $\mathrm{N}_{2} \mathrm{O}$ emission factors which are essential in improving national and regional GHG inventories.
\end{abstract}

\section{Introduction}

Nitrous oxide $\left(\mathrm{N}_{2} \mathrm{O}\right)$, a potent greenhouse gas $(\mathrm{GHG})$ with a global warming potential (GWP) of 265 compared to carbon dioxide $\left(\mathrm{CO}_{2}\right)$, has attracted much attention globally [1]. Its global concentration in the atmosphere has been rising (estimated at $\sim 331.1 \mathrm{ppb}$ ) and contributes about $6 \%$ of the GHG-caused global warming effect [2]. Increased $\mathrm{N}_{2} \mathrm{O}$ concentration has increased average atmospheric temperature causing global warming, associated with unreliable precipitation and droughts [3]. Prolonged droughts result in crop failures while unpredictable rainfall inconveniences 
cropping calendar, especially planting schedule for rainfed smallholder farming, prompting food insecurity [4]. Besides its adverse effects on climate variability, $\mathrm{N}_{2} \mathrm{O}$ is also associated with stratospheric ozone layer depletion and acid rain formation [5, 6]. Assessment of agricultural contribution to soil $\mathrm{N}_{2} \mathrm{O}$ fluxes is essential for climate change mitigation.

Agriculture is a significant source of anthropogenic nitrous oxide $\left(\mathrm{N}_{2} \mathrm{O}\right)$ emissions, contributing about $60 \%$ of global $\mathrm{N}_{2} \mathrm{O}$ emissions predominantly from $\mathrm{N}$ fertilizers, animal manure, and crop residues left in fields $[7,8]$. In SSA, agriculture covers about $12.6 \%$ of total cultivated land, dominated by smallholder farmers who produce crops depending on resource availability [9]. Over 95\% of agricultural land in SSA is rainfed, nonmechanized, and under small-scale farming with inherent low fertility due to continuous farming with limited use of external soil inputs Altieri and Koohafkan[10] which could have a direct effect on the amounts of soil $\mathrm{N}_{2} \mathrm{O}$ emitted [11]. These agricultural soils in SSA contribute between $6 \%$ and $19 \%$ of total global anthropogenic $\mathrm{N}_{2} \mathrm{O}$ emissions $[12,13]$. However, literature on the contribution of different cropping systems on soil $\mathrm{N}_{2} \mathrm{O}$ fluxes in SSA is limited.

Different cropping systems exist across various regions in SSA. The common crops grown in West Africa, Southern Africa, and Central Africa are cassava, yams, and cereals such as maize and sorghum [14]. With perennial cropping systems, maize is common in Eastern and part of Southern Africa [15]. Different dynamics across cropping systems contribute differently to $\mathrm{N}_{2} \mathrm{O}$ emissions $[14,16]$. For instance, cereal-legume intercropping contribute to $\mathrm{N}_{2} \mathrm{O}$ emissions through the addition of more $\mathrm{NH}_{4}{ }^{+}$and $\mathrm{NO}_{3}{ }^{-}$ into soils from mineralization of organic matter $[17,18]$. Additionally, farmers in SSA integrate livestock and crops leading to a trade-off between manure and crop residues, a dynamic that influences $\mathrm{N}$ cycling (including $\mathrm{N}_{2} \mathrm{O}$ ) in soils $[19,20]$. However, most smallholder farmers in SSA rarely retain crop residues on fields as sources of nutrients but instead use them as animal feeds and as fuel for cooking [21].

Nitrogen undergoes a complex biogeochemical process (Figure 1). First, atmospheric nitrogen is biologically fixed by leguminous plants associated with rhizobia bacteria and nitrogenase enzymes [22]. Second, human-induced activities such as the production of fertilizer, sewage, farm produce, and manure application also account for $\mathrm{N}$ addition into the soil, which is later released as $\mathrm{N}_{2} \mathrm{O}$ emissions (Figure 1). Further, high $\mathrm{N}$ accumulation in the soil is associated with environmental problems such as ammonia volatilization and leaching, also indirect losses of $\mathrm{N}$ from soils. Net $\mathrm{N}_{2} \mathrm{O}$ emissions result from complex biogeochemical processes that take place in soils [23]. Nitrification occurs during aerobic conditions and oxidizes ammonium $\left(\mathrm{NH}_{4}{ }^{+}\right)$to nitrate $\left(\mathrm{NO}_{3}{ }^{-}\right)$and nitrite $\left(\mathrm{NO}_{2}\right)$ [24]. Additionally, denitrification occurs in oxygen-limited situations and reduces $\mathrm{NO}_{3}{ }^{-}$and $\mathrm{NO}_{2}{ }^{-}$to $\mathrm{N}_{2} \mathrm{O}$ and nitrogen gas $\left(\mathrm{N}_{2}\right)$ [25]. These processes are aided by the availability of three significant microorganisms: ammonia-oxidizing bacteria (AOA), ammonia-oxidizing archaea (AOA), and nitrite-oxidizing bacteria (NOB) [26]. Nitrous oxide emissions can also occur through nitrate reduction to ammonium and codenitrification [27]. Other nonbiological processes involved in $\mathrm{N}_{2} \mathrm{O}$ emissions are chemodenitrification and hydroxylamine decomposition, although they release negligible $\mathrm{N}_{2} \mathrm{O}$ emissions [28]. These processes are influenced by soil moisture, temperature, $\mathrm{C} / \mathrm{N}$ ratio, oxygen concentration, organic carbon, and soil nitrogen availability [11].

Developing countries are obligated to report their $\mathrm{Na}$ tionally Determined Contributions (NDCs) and climate change mitigation options to the United Nations Framework Convention on Climate Change (UNFCCC) [29, 30]. Further, as captured in the Paris Climate Agreement of 2015, countries agreed to limit global temperature increase below $1.5^{\circ} \mathrm{C}$ by reducing $\mathrm{GHG}$ emissions [31]. To achieve this, most SSA countries consider agriculture a potential mitigation option to reduce GHG emissions [32]. However, there are uncertainties in national GHG inventories in SSA countries. A vast data gap arising from countries in the region has limited, or none whatsoever, empirical studies from existing cropping systems. It is imperative to note that only a few studies in SSA (approximately 30 published studies) have attempted to quantify $\mathrm{N}_{2} \mathrm{O}$ emissions based on different cropping systems (Figure 2).

Consequently, most of the countries in SSA have continuously used Intergovernmental Panel on Climate Change (IPCC) Tier 1 emission factors (EFs), which tend to overestimate GHG emissions in the region [21, 32, 33]. Therefore, in this paper, we reviewed the state of $\mathrm{N}_{2} \mathrm{O}$ emissions from selected cropping systems, drivers that significantly influence $\mathrm{N}_{2} \mathrm{O}$ emissions, and probable soil $\mathrm{N}_{2} \mathrm{O}$ emissions mitigation options in SSA cropping systems. We hypothesized that, in SSA, (i) there are significant variations in $\mathrm{N}_{2} \mathrm{O}$ emissions across different cropping systems, (ii) environmental factors, climatic conditions, farm management practices, and soil properties directly influence $\mathrm{N}_{2} \mathrm{O}$ dynamics, and (iii) combination of inorganic and organic fertilizer application serves as best mitigation options for $\mathrm{N}_{2} \mathrm{O}$ emissions compared to sole application of either organic or inorganic fertilizer.

\section{Methodology}

Our literature review surveyed peer-reviewed papers on $\mathrm{N}_{2} \mathrm{O}$ fluxes from Sub-Saharan Africa cropping systems published until December 2020. To include as many published studies as possible, we used search terms such as "nitrous oxide," "Sub-Saharan Africa," "cropping systems," "greenhouse gas emission," "nitrous oxide yield-scaled emissions," "nitrous oxide emission factors," and "mitigation measures" in Web of Science and Google Scholar. Thirty (30) peer-reviewed papers were selected according to the following exclusion-inclusion criteria:

(1) The study measured nitrous oxide fluxes in situ in Sub-Saharan Africa.

(2) The static chamber method was used in nitrous oxide measurements.

(3) Nitrous oxide measurements were conducted from a specified period. 


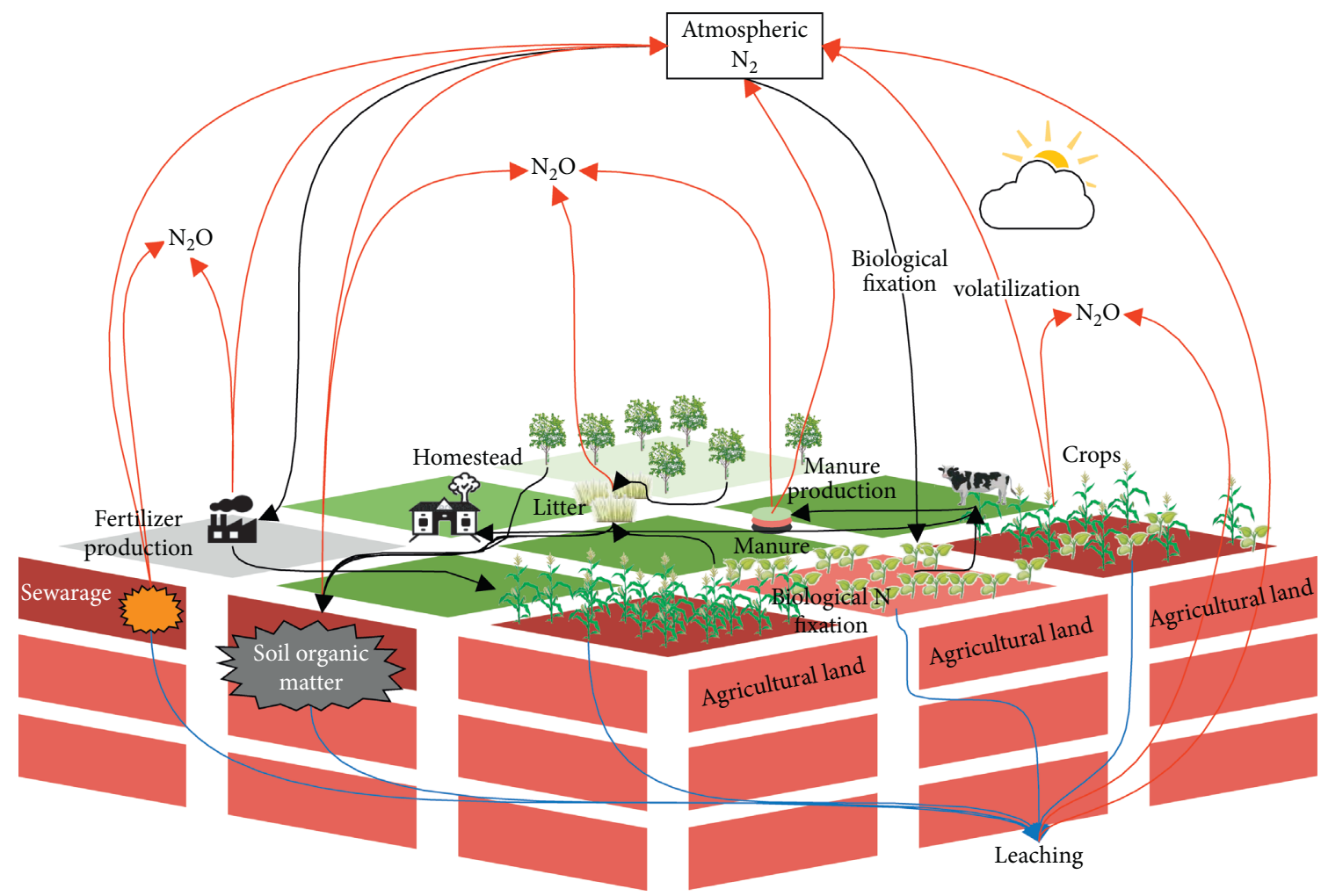

Figure 1: Nitrogen transformation processes in soil. The arrows in red show $\mathrm{N}_{2} \mathrm{O}$ emissions, ones in black are $\mathrm{N}_{2} \mathrm{O}$ sources, and blue ones are $\mathrm{N}$ losses.

(4) The study reported nitrous oxide fluxes and either yield, $\mathrm{N}_{2} \mathrm{O}$ emission factors, yield-scaled emission, or mitigation potential.

(5) Soil properties, cropping system, soil fertility management, experimental durations were clearly described.

A qualitative analysis was implemented to assess nitrous oxide fluxes, $\mathrm{N} 2 \mathrm{O}$ emission factors, yield-scaled $\mathrm{N}_{2} \mathrm{O}$ emission factors, and mitigation potential. This included reporting the data observed from different cropping systems in Sub-Saharan Africa.

\section{Soil $\mathbf{N}_{2} \mathbf{O}$ Emissions from Cropping Systems in Sub-Saharan Africa}

3.1. Maize Cropping System. Most of the soil $\mathrm{N}_{2} \mathrm{O}$ quantification experiments carried out in SSA are under maize cropping systems (Table 1). This is because maize is considered an important food and source of cash for most rural families in the region [60]. Millar et al. [50] reported $\mathrm{N}_{2} \mathrm{O}$ $\left(\mathrm{N}_{2} \mathrm{O}-\mathrm{N}\right)$ emissions ranging between 0.1 and $4.1 \mathrm{~kg} \mathrm{ha}^{-1}$ from maize cropping systems in Kenya under improvedfallow agroforestry systems (Table 1). While investigating the effects of organic and mineral fertilizers in Zimbabwe, Mapanda et al. [52] reported $\mathrm{N}_{2} \mathrm{O}$ emissions ranging between 0.1 and $0.5 \mathrm{~kg} \mathrm{ha}^{-1}$. Moreover, while evaluating the effects of clearing savannah woodland for maize cropping in Zimbabwe, Mapanda et al. [38] reported 0.8 to
$2.5 \mathrm{~kg} \mathrm{~N}_{2} \mathrm{O}-\mathrm{N} \mathrm{ha}^{-1}$ (Table 1). Hickman et al. [41] studied the relationship between $\mathrm{N}$ inputs and $\mathrm{N}_{2} \mathrm{O}$ emissions from maize cropping systems in Kenya and reported $\mathrm{N}_{2} \mathrm{O}-\mathrm{N}$ fluxes ranging between 0.1 and $0.3 \mathrm{~kg} \mathrm{ha}^{-1} \mathrm{yr}^{-1}$ (Table 1). Further, with no fertilizer or manure application, Rosenstock et al. [42] reported $\mathrm{N}_{2} \mathrm{O}$ emissions from maize cropping systems as $0.9 \mathrm{~kg} \mathrm{ha}^{-1} \mathrm{yr}^{-1}$ in Kolero, Tanzania (Table 1).

Pelster et al. [1] reported that maize cropping systems with low fertilizer inputs $\left(<25 \mathrm{~kg} \mathrm{~N} \mathrm{ha}^{-1}\right)$ were responsible for $\mathrm{N}_{2} \mathrm{O}$ fluxes ranging between -0.1 and $1.8 \mathrm{~kg} \mathrm{ha}^{-1} \mathrm{yr}^{-1}$ in Kenya, attributing observed $\mathrm{N}_{2} \mathrm{O}$ fluxes to dry soil which limits anaerobic condition for denitrification (Table 1). While investigating $\mathrm{N}_{2} \mathrm{O}$ emissions from different inorganic fertilizer rates and their combination with an organic fertilizer in Zimbabwe under maize cropping systems, Nyamadzawo et al. [53] reported $\mathrm{N}_{2} \mathrm{O}$ emissions ranging from 0.3 to $0.5 \mathrm{~kg} \mathrm{ha}^{-1} \mathrm{yr}^{-1}$. Additionally, while studying the contribution of different soil fertility technologies towards the national GHG budget in the central highlands of Kenya, Macharia et al. [28] reported 0.13 to $1.22 \mathrm{~kg} \mathrm{~N}_{2} \mathrm{O}-\mathrm{N} \mathrm{ha}^{-1} \mathrm{yr}^{-1}$ across treatments. Similarly, Musafiri et al. [48] also reported $\mathrm{N}_{2} \mathrm{O}$ emissions ranging from 0.21 to $0.38 \mathrm{~kg} \mathrm{~N}_{2} \mathrm{O}-\mathrm{N} \mathrm{ha} \mathrm{yr}^{-1}$ under the maize cropping system. Hence, we note that maize cropping systems emit less $\mathrm{N}_{2} \mathrm{O}$ emissions than the global average, probably due to soil degradation and soil $\mathrm{N}$ mining alongside inadequate nutrient replenishment from external inputs [61]. 


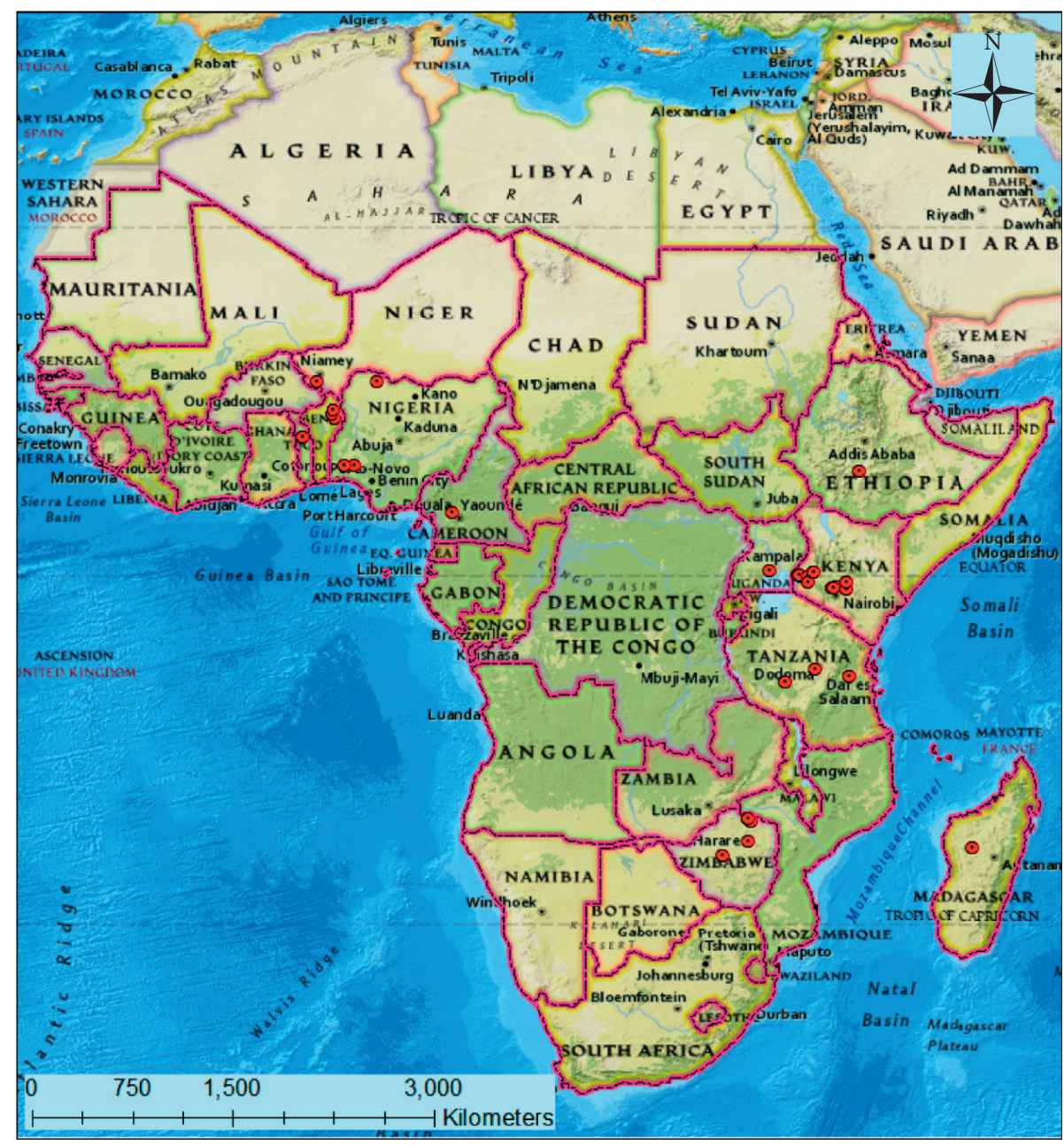

- Reviewed studies

SSA countries boundaries

Figure 2: Map showing the location of reviewed $\mathrm{N}_{2} \mathrm{O}$ related studies in Sub-Saharan countries. Basemap sources: National Geographic, Esri, Garmin, HERE, UNEP, WCMC, USGS, NASA, ESA, MERI, NRCAN, GEBCO, NOAA, and increment P Corp.

3.2. Cereal-Legume Intercropping/Rotation System. Legume-cereal intercropping is a farming practice that acts as an $\mathrm{N}$ source through atmospheric $\mathrm{N}$ fixation [62]. However, the addition of $\mathrm{N}$ in the soils can come at the cost of increased $\mathrm{N}_{2} \mathrm{O}$ emissions if supply exceeds plant demand [41]. For example, Millar et al. [50] recorded $4.1 \mathrm{~kg} \mathrm{ha}^{-1} \mathrm{~N}_{2} \mathrm{O}$ emissions from a maize-bean intercropping system in Kenya following the incorporation of Sesbania and Macroptilium. These fluxes were the highest recorded in SSA, which could be attributed to residue application with $60 \%$ more $\mathrm{N}$ content above the normal threshold $(1.7 \%$ to $1.8 \%)$ [63]. From a short-term experiment in Western Kenya, Baggs et al. [9], working on effects of tillage and residue quality on GHG emissions under an improved-fallow agroforestry system, showed that maize (Zea mays) intercropped with beans (Phaseolus vulgaris) emits 0.2 to $0.6 \mathrm{~kg} \mathrm{~N}_{2} \mathrm{O} \mathrm{ha}^{-1}$. Rotation of millet and beans in Mali accounted for $\mathrm{N}_{2} \mathrm{O}$ emissions that ranged from 0.9 to $1.5 \mathrm{~kg} \mathrm{ha}^{-1} \mathrm{yr}^{-1}$ [64]. Ortiz-Gonzalo et al. [43] showed that maize (Zea mays) intercropped with beans (Phaseolus vulgaris) in central highlands of Kenya emitted $\mathrm{N}_{2} \mathrm{O}$ in the range of 0.18 to $0.27 \mathrm{~kg} \mathrm{ha}^{-1}$. Inorganically fertilized maize crops intercropped with lablab (L. purpureus) and Crotalaria (C. juncea) in Ethiopia emitted between 0.17 and $0.33 \mathrm{~kg} \mathrm{~N}_{2} \mathrm{O}-\mathrm{N} \mathrm{ha} \mathrm{hr}^{-1}$ [47]. Intercropping or rotating cereals with legumes provides synergies in managing soil nutrients in the field and may result in relatively lower $\mathrm{N}_{2} \mathrm{O}$ emissions.

3.3. Coffee Cropping System. Coffee is among the critical annual cash crops grown in SSA. In Kenya, Ortiz-Gonzalo et al. [43] reported $\mathrm{N}_{2} \mathrm{O}$ emissions that ranged between 1 and $1.9 \mathrm{~kg} \mathrm{~N}_{2} \mathrm{O}-\mathrm{N} \mathrm{ha}^{-1} \mathrm{yr}^{-1}$ from coffee cropping system following fertilizer application of $85 \mathrm{~kg} \mathrm{Nha}^{-1}$ (Table 1). In Tanzania, Gütlein et al. [65] established that coffee cropping systems accounted for $0.35 \mathrm{~kg} \mathrm{~N}_{2} \mathrm{O}-\mathrm{N} \mathrm{ha}^{-1} \mathrm{yr}^{-1}$. Soil $\mathrm{N}_{2} \mathrm{O}$ emissions from coffee cropping systems in SSA are at a lower range, probably due to a decline in soil fertility in SSA's soil and little nutrient supply from both organics and inorganics [65]. 
TABLE 1: In situ empirical studies on $\mathrm{N}_{2} \mathrm{O}$ emissions from different cropping systems in Sub-Saharan Africa.

\begin{tabular}{|c|c|c|c|c|c|c|c|c|}
\hline $\begin{array}{l}\text { Cropping } \\
\text { systems }\end{array}$ & Country & $\begin{array}{l}\text { Sampling } \\
\text { frequency }\end{array}$ & $\begin{array}{l}\text { Sampling } \\
\text { duration }\end{array}$ & Soil type & $\mathrm{N}_{2} \mathrm{O}$ fluxes & $\begin{array}{c}\mathrm{N}_{2} \mathrm{O} \text { EFs } \\
(\%)\end{array}$ & $\begin{array}{c}\mathrm{N}_{2} \mathrm{O} \text { YSE (g } \\
\mathrm{N}_{2} \mathrm{O} \mathrm{kg} \\
\text { grain) }\end{array}$ & References \\
\hline \multicolumn{9}{|c|}{ Annual emissions } \\
\hline $\begin{array}{l}\text { Sorghum, } \\
\text { peanut, } \\
\text { and } \\
\text { groundnut }\end{array}$ & $\begin{array}{l}\text { Burkina } \\
\text { Faso }\end{array}$ & 1-3 per week & Jun-Sep 2006 & Sandy & $0.19-0.67 \mathrm{~kg} \mathrm{ha}^{-1} \mathrm{yr}^{-1}$ & $*$ & $*$ & $\begin{array}{c}\text { Brummer et al. } \\
{[34]}\end{array}$ \\
\hline $\begin{array}{l}\text { Millet- } \\
\text { legume } \\
\text { intercrop }\end{array}$ & Mali & Monthly & $\begin{array}{c}\text { Jan } 2004-F e b \\
2005\end{array}$ & Alfisol & $0.9-1.5 \mathrm{~kg} \mathrm{ha}^{-1} \mathrm{yr}^{-1}$ & 4.1 & $*$ & Dick et al. [35] \\
\hline Vegetable & Niger & $\begin{array}{l}\text { Twice a day } \\
\text { for } 6 \text { days }\end{array}$ & $\begin{array}{c}\text { Apr } \\
2006-\mathrm{Feb} \\
2007\end{array}$ & Sandy & $48-92 \mathrm{~kg} \mathrm{ha}^{-1} \mathrm{yr}^{-1}$ & $*$ & $*$ & $\begin{array}{l}\text { Predotova } \\
\text { et al. [36] }\end{array}$ \\
\hline Vegetable & Zimbabwe & Twice a day & $\begin{array}{c}\text { Mar } \\
\text { 2008-Mar } \\
2009\end{array}$ & Clay & $80.5-113.4 \mathrm{~kg} \mathrm{ha}^{-1} \mathrm{yr}^{-1}$ & $3-4$ & & $\begin{array}{l}\text { Lompo et al. } \\
\text { [37] }\end{array}$ \\
\hline Maize & Zimbabwe & $\begin{array}{l}\text { During } \\
\text { raining season }\end{array}$ & $\begin{array}{l}\text { Jun } \\
\text { 2006-May } \\
2009\end{array}$ & Clay/loam & $0.8-2.5 \mathrm{~kg} \mathrm{ha}^{-1} \mathrm{yr}^{-1}$ & & & $\begin{array}{c}\text { Mapanda et al. } \\
{[38]}\end{array}$ \\
\hline Fruit & Zimbabwe & $\begin{array}{c}2 \\
\text { days-2 weeks }\end{array}$ & 2011-2013 & Sandy loam & $2.5-112 \mathrm{~kg} \mathrm{ha}^{-1}$ & $*$ & $2.1-14$ & $\begin{array}{l}\text { Nyamadzawo } \\
\text { et al. [39] }\end{array}$ \\
\hline Maize & Kenya & $\begin{array}{l}\text { Daily to } \\
\text { weekly }\end{array}$ & 99 days & Clay & $0.16-0.81 \mathrm{~kg} \mathrm{ha}^{-1} \mathrm{yr}^{-1}$ & $*$ & $*$ & $\begin{array}{c}\text { Hickman et al. } \\
{[40]}\end{array}$ \\
\hline Maize & Kenya & $\begin{array}{l}\text { Daily to } \\
\text { weekly }\end{array}$ & $\begin{array}{l}\text { March } \\
\text { 2011-July } \\
2011\end{array}$ & Sandy-clay & $0.1-0.3 \mathrm{~kg} \mathrm{ha}^{-1} \mathrm{yr}^{-1}$ & 0.11 & $0.27-0.8$ & $\begin{array}{c}\text { Hickman et al. } \\
{[41]}\end{array}$ \\
\hline $\begin{array}{l}\text { Tea, } \\
\text { vegetable, } \\
\text { and maize }\end{array}$ & Kenya & Weekly & Jan-Dec 2013 & Sand-clay & $0.4-3.9 \mathrm{~kg} \mathrm{ha}^{-1} \mathrm{yr}^{-1}$ & $0.4-0.8$ & * & $\begin{array}{l}\text { Rosenstock } \\
\text { et al. [42] }\end{array}$ \\
\hline $\begin{array}{l}\text { Maize, } \\
\text { beans, and } \\
\text { sorghum }\end{array}$ & Kenya & Weekly & $\begin{array}{c}\text { Aug } \\
\text { 2013-Aug } \\
2014\end{array}$ & Nitisols & $-0.1-1.8 \mathrm{~kg} \mathrm{ha}^{-1} \mathrm{yr}^{-1}$ & $*$ & $1.1-67$ & $\begin{array}{c}\text { Pelster et al. } \\
{[1]}\end{array}$ \\
\hline $\begin{array}{l}\text { Maize, } \\
\text { bean, and } \\
\text { coffee }\end{array}$ & Kenya & $\begin{array}{l}\text { 1-2 times a } \\
\text { week }\end{array}$ & $\begin{array}{c}\text { Feb } 2015 \\
\text {-Feb } 2016\end{array}$ & Nitisols & $1-1.9 \mathrm{~kg} \mathrm{ha}^{-1} \mathrm{yr}^{-1}$ & $<1$ & $0.08-0.15$ & $\begin{array}{l}\text { Ortiz-Gonzalo } \\
\text { et al. [43] }\end{array}$ \\
\hline Tea & Kenya & $\begin{array}{l}\text { 1-2 times per } \\
\text { week }\end{array}$ & $\begin{array}{l}\text { Aug } \\
\text { 2015-July } \\
2016\end{array}$ & $\begin{array}{l}\text { Humic } \\
\text { nitisols }\end{array}$ & $0.6-2.34 \mathrm{~kg} \mathrm{ha}^{-1} \mathrm{yr}^{-1}$ & $*$ & $*$ & $\begin{array}{l}\text { Wanyama } \\
\text { et al. [44] }\end{array}$ \\
\hline Maize & Ghana & $\begin{array}{l}\text { Daily during } \\
\text { fertilization, } \\
\text { then weekly }\end{array}$ & $\begin{array}{c}\text { Aug } \\
\text { 2013-Aug } \\
2014\end{array}$ & $\begin{array}{l}\text { Ferric } \\
\text { luvisol }\end{array}$ & $1.22-4.29 \mathrm{~kg} \mathrm{ha}^{-1} \mathrm{yr}^{-1}$ & $0.1-0.55$ & $0.39-1.24$ & $\begin{array}{c}\text { Atakora et al. } \\
{[45]}\end{array}$ \\
\hline Maize & Tanzania & $\begin{array}{l}\text { Weekly to } \\
\text { monthly }\end{array}$ & $\begin{array}{l}\text { Dec 2015- } \\
\text { Nov } 2017\end{array}$ & $\begin{array}{l}\text { Alfisol/ } \\
\text { andisol }\end{array}$ & $0.26-2.24 \mathrm{~kg} \mathrm{ha}^{-1} \mathrm{yr}^{-1}$ & $0.1-1.3$ & 0.18 & $\begin{array}{l}\text { Zheng et al. } \\
{[46]}\end{array}$ \\
\hline $\begin{array}{l}\text { Maize- } \\
\text { legumes } \\
\text { (lablab/ } \\
\text { Crotalaria }\end{array}$ & Ethiopia & Weekly & $107-123$ days & Clay-loam & $0.17-0.33 \mathrm{~kg} \mathrm{ha}^{-1} \mathrm{yr}^{-1}$ & $0.2-0.25$ & & $\begin{array}{c}\text { Raji and } \\
\text { Dörsch [47] }\end{array}$ \\
\hline Maize & Kenya & Weekly & $\begin{array}{c}\text { Feb 2017-Feb } \\
2018\end{array}$ & Sandy loam & $0.13-1.22 \mathrm{~kg} \mathrm{ha}^{-1} \mathrm{yr}^{-1}$ & $0.2-0.9$ & $0.5-2.2$ & $\begin{array}{c}\text { Macharia et al. } \\
{[28]}\end{array}$ \\
\hline Maize & Kenya & Weekly & $\begin{array}{c}\text { March } \\
\text { 2018-March } \\
2019\end{array}$ & $\begin{array}{l}\text { Humic } \\
\text { nitisols }\end{array}$ & $0.21-0.38 \mathrm{~kg} \mathrm{ha}^{-1} \mathrm{yr}^{-1}$ & $0.05-1.4$ & $0.024-0.028$ & $\begin{array}{c}\text { Musafiri et. } \\
\text { [48] }\end{array}$ \\
\hline \multicolumn{9}{|c|}{ Seasonal fluxes } \\
\hline Maize & Zimbabwe & Weekly & $\begin{array}{c}\text { Dec } \\
2000-F e b \\
2001\end{array}$ & Sandy loam & $0.1-0.3 \mathrm{~kg} \mathrm{ha}^{-1}$ & 0.2 & $*$ & $\begin{array}{c}\text { Chikowo et al. } \\
\text { [49] }\end{array}$ \\
\hline Maize & Kenya & $\begin{array}{l}\text { 1-2 times per } \\
\text { week }\end{array}$ & 84 days & Sandy-clay & $0.1-4.1 \mathrm{~kg} \mathrm{ha}^{-1}$ & $*$ & $*$ & $\begin{array}{l}\text { Millar et al. } \\
{[50]}\end{array}$ \\
\hline Maize & Kenya & Weekly & $\begin{array}{l}\text { Feb-June } \\
2002\end{array}$ & $\begin{array}{l}\text { Silt-clay- } \\
\text { loam }\end{array}$ & $0.2-0.6 \mathrm{~kg} \mathrm{ha}^{-1}$ & $*$ & $*$ & $\begin{array}{l}\text { Baggs et al. } \\
{[18]}\end{array}$ \\
\hline
\end{tabular}


TABle 1: Continued.

\begin{tabular}{|c|c|c|c|c|c|c|c|c|}
\hline $\begin{array}{l}\text { Cropping } \\
\text { systems }\end{array}$ & Country & $\begin{array}{l}\text { Sampling } \\
\text { frequency }\end{array}$ & $\begin{array}{l}\text { Sampling } \\
\text { duration }\end{array}$ & Soil type & $\mathrm{N}_{2} \mathrm{O}$ fluxes & $\begin{array}{c}\mathrm{N}_{2} \mathrm{O} \text { EFs } \\
(\%)\end{array}$ & $\begin{array}{c}\mathrm{N}_{2} \mathrm{O} \text { YSE (g } \\
\mathrm{N}_{2} \mathrm{O} \mathrm{kg} \\
\text { grain) }\end{array}$ & References \\
\hline $\begin{array}{l}\text { Soybeans } \\
\text { and maize }\end{array}$ & Madagascar & Weekly & $\begin{array}{c}\text { Nov } \\
\text { 2006-Apr } \\
2007\end{array}$ & Ferralsol & $0.3 \mathrm{~kg} \mathrm{ha}^{-1}$ & $0.46-0.47$ & $*$ & $\begin{array}{c}\text { Chapuis- } \\
\text { Lardy et al. } \\
{[51]}\end{array}$ \\
\hline Maize & Zimbabwe & $\begin{array}{l}\text { Once every } \\
\text { two months }\end{array}$ & $\begin{array}{c}\text { Jan } \\
\text { 2006-May } \\
2009\end{array}$ & $\begin{array}{l}\text { Clay and } \\
\text { sandy loam }\end{array}$ & $0.1-0.5 \mathrm{~kg} \mathrm{ha}^{-1}$ & $*$ & $0.02-3.93$ & $\begin{array}{c}\text { Mapanda et al. } \\
\text { [52] }\end{array}$ \\
\hline Maize & Zimbabwe & & $\begin{array}{l}2008 / 2009 \\
\text { growing } \\
\text { season }\end{array}$ & Sandy loam & $0.26-0.52 \mathrm{~kg} \mathrm{ha}^{-1}$ & $*$ & $0.22-0.68$ & $\begin{array}{c}\text { Nyamadzawo } \\
\text { et al. [53] }\end{array}$ \\
\hline Vegetable & Kenya & $\begin{array}{l}\text { 1-3 days per } \\
\text { week }\end{array}$ & $\begin{array}{c}\text { Sept } \\
\text { 2015-July } \\
2016\end{array}$ & $\begin{array}{l}\text { Humic } \\
\text { nitisols }\end{array}$ & $0.4-3.0 \mathrm{~kg} \mathrm{ha}^{-1}$ & $0.0-2.6$ & $*$ & $\begin{array}{c}\text { Kurgat et al. } \\
{[54]}\end{array}$ \\
\hline $\begin{array}{l}\text { Coffee, } \\
\text { maize- } \\
\text { beans }\end{array}$ & Kenya & $\begin{array}{l}\text { 1-3 days per } \\
\text { week }\end{array}$ & $\begin{array}{l}\text { Feb 2015- } \\
\text { Feb } 2016\end{array}$ & Nitisols & $0.18-1.9 \mathrm{~kg} \mathrm{ha}^{-1}$ & & & $\begin{array}{l}\text { Ortiz-Gonzalo } \\
\text { et al. [43] }\end{array}$ \\
\hline \multicolumn{9}{|c|}{ Short duration } \\
\hline $\begin{array}{l}\text { Maize- } \\
\text { beans }\end{array}$ & Nigeria & $\begin{array}{l}1-3 \text { days to } \\
2 \text { weeks }\end{array}$ & 21 days & $\begin{array}{l}\text { Ferric } \\
\text { lixisol }\end{array}$ & $0.1-0.3 \mathrm{~kg} \mathrm{~N} \mathrm{ha}^{-1} /$ day & 1 & & $\begin{array}{l}\text { Roing et al. } \\
\text { [55] }\end{array}$ \\
\hline Maize & Kenya & $\begin{array}{l}3 \text { times per } \\
\text { month }\end{array}$ & 4 weeks & $\begin{array}{l}\text { Humic } \\
\text { nitisols }\end{array}$ & $1.3-12 \mu \mathrm{g} \mathrm{m}^{-2} \mathrm{~h}^{-1}$ & $*$ & $*$ & $\begin{array}{c}\text { Kimetu et al. } \\
{[56]}\end{array}$ \\
\hline Vegetables & Zimbabwe & Biweekly & $\begin{array}{c}\text { Sept } \\
2007-\text { Nov } \\
2008\end{array}$ & $\begin{array}{l}\text { Loamy } \\
\text { sandy }\end{array}$ & $2.5-18.8 \mathrm{~g} \mathrm{~N}_{2} \mathrm{O}-\mathrm{N} \mathrm{h}^{-1}$ & $0.3-1.0$ & $*$ & $\begin{array}{c}\text { Masaka et al. } \\
\text { [57] }\end{array}$ \\
\hline $\begin{array}{l}\text { Banana- } \\
\text { coffee }\end{array}$ & Uganda & $\begin{array}{l}\text { 4-5 times per } \\
\text { month }\end{array}$ & $\begin{array}{c}\text { May } \\
\text { 2018-June } \\
2018\end{array}$ & $\begin{array}{l}\text { Sandy-clay } \\
\text { loam }\end{array}$ & $3.7-6.7 \mu \mathrm{g} \mathrm{m}^{-2} \mathrm{~h}^{-1}$ & & $*$ & $\begin{array}{c}\text { Fatumah et al. } \\
{[58]}\end{array}$ \\
\hline Amaranth & $\begin{array}{l}\text { Benin/ } \\
\text { Nigeria }\end{array}$ & $\begin{array}{l}\text { Daily during } \\
\text { planting and } \\
\text { then after } 2 \\
\text { weeks }\end{array}$ & 21 days & $\begin{array}{l}\text { Haplic } \\
\text { lixisols/ } \\
\text { plethnic } \\
\text { plinthosols }\end{array}$ & $\begin{array}{l}24.8-279.5 \mathrm{mgN} / \mathrm{kg} \\
\text { soil }\end{array}$ & & & $\begin{array}{l}\text { Olaleye et al. } \\
\text { [59] }\end{array}$ \\
\hline
\end{tabular}

N/B. All measurements were carried out using static chambers. $*$ no value reported.

3.4. Tea Cropping System. Tea cropping systems are precious and primarily found in agroecological zones that receive high rainfall amounts. Rosenstock et al. [42] showed that tea cropping systems in western Kenya emitted $\mathrm{N}_{2} \mathrm{O}$ fluxes that ranged between 0.4 and $0.7 \mathrm{~kg} \mathrm{~N}_{2} \mathrm{O}-\mathrm{N}$ ha ${ }^{-1}$. Tea cropping systems in Kenya emitted between 1.2 and $1.4 \mathrm{~kg} \mathrm{~N}_{2} \mathrm{O}-\mathrm{N}$ $\mathrm{ha}^{-1} \mathrm{yr}^{-1}$ [44]. Emissions of $\mathrm{N}_{2} \mathrm{O}$ for tea cropping systems were also at a lower range attributed to low inherent soil fertility status with little or no replenishment with soil amendments.

3.5. Vegetable Cropping System. Due to high amounts of organic or inorganic fertilization, vegetable cropping systems produce the highest $\mathrm{N}_{2} \mathrm{O}$ emissions across different cropping systems in SSA. For instance, vegetable cropping systems in Niger had $\mathrm{N}_{2} \mathrm{O}$ emissions ranging between 48 and $92 \mathrm{~kg} \mathrm{ha}^{-1} \mathrm{yr}^{-1}$ [36]. Peri-urban vegetable gardens in Burkina Faso emitted $\mathrm{N}_{2} \mathrm{O}$ emissions that ranged from 80.5 to $113.4 \mathrm{~kg} \mathrm{ha}^{-1} \mathrm{yr}^{-1}$ [37]. Cumulative annual vegetable $\mathrm{N}_{2} \mathrm{O}$ fluxes in Kaptumo, Kenya, were found to be $0.9 \mathrm{~kg} \mathrm{~N}_{2} \mathrm{O}-\mathrm{N}$ $\mathrm{ha}^{-1} \mathrm{yr}^{-1}$ [42]. Similarly, in Kenya, indigenous vegetables produced 0.4 to $3.0 \mathrm{~kg} \mathrm{~N}_{2} \mathrm{O}-\mathrm{N} \mathrm{ha}^{-1}$ [54]. While comparing GHG emissions in two ecoregions of SSA, Benin (rain forest) and Nigeria (dry savannah), in a short experiment performed under controlled conditions under local amaranth (Amaranthus cruentus), Olaleye et al. [59] reported $\mathrm{N}_{2} \mathrm{O}$ emissions ranging from 0.01 to 0.02 and 0.06 to $0.3 \mathrm{~kg} \mathrm{~N} \mathrm{~kg}^{-1}$ of soil day ${ }^{-1}$, respectively. There was a high variation of $\mathrm{N}_{2} \mathrm{O}$ emissions ( 0.01 to $113 \mathrm{~kg} \mathrm{Nha}^{-1} \mathrm{yr}^{-1}$ ) for vegetable cropping systems. The variation was attributed to high $\mathrm{N}$ input ( 25 to $750 \mathrm{~kg} \mathrm{Nha}^{-1}$ ) in vegetable gardens.

3.6. $\mathrm{N}_{2} \mathrm{O}$ Emissions from Organic and Inorganic Fertilizer Use. Organic resources in SSA improve soil fertility and overall soil health and increase crop yields [66]. However, the addition of organic resources may contribute to increased soil $\mathrm{N}_{2} \mathrm{O}$ emissions [66]. Some of the organic inputs used in SSA include animal manure, Tithonia diversifolia, numerous leguminous plants, crop residues, and some herb trees such as Lantana Camara $[25,67,68]$. A couple of studies have quantified organic resources' effects on $\mathrm{N}_{2} \mathrm{O}$ emissions in SSA across cropping systems. The addition of Tithonia diversifolia increased $\mathrm{N}_{2} \mathrm{O}$ emissions, especially during the first weeks of application, as shown by Kimetu et al. [56], implying that organic matter decomposes rapidly in soil. Green manure quickly releases nutrients to the soil immediately after addition since they contain easily decomposable organic matter for microorganism consumption as 
substrates [69]. The use of cattle manure as a treatment in tomatoes production planted in a wetland in Zimbabwe led to 0.01 to $0.06 \mathrm{~kg} \mathrm{~N}_{2} \mathrm{O}-\mathrm{N} \mathrm{ha}^{-1}$ emissions [70]. Higher $\mathrm{N}_{2} \mathrm{O}$ fluxes of $43 \mu \mathrm{g} \mathrm{N}_{2} \mathrm{O}-\mathrm{N} \mathrm{m}^{-2} \mathrm{~h}^{-1}$ were observed in Central Highlands of Kenya under manure treatment compared with $3 \mu \mathrm{g} \mathrm{N}_{2} \mathrm{O}-\mathrm{Nm}^{-2} \mathrm{~h}^{-1}$ under no external inputs [43]. While investigating GHGs emissions from maize cropping systems under different soil fertility management, Macharia et al. [28] showed that plots treated with animal manure had $1.22 \mathrm{~kg} \mathrm{~N}_{2} \mathrm{O}-\mathrm{N} \mathrm{ha}{ }^{-1} \mathrm{yr}^{-1}$, ninefold higher than the control treatment.

Nitrogen-based inorganic fertilizer application significantly affects the amount of soil $\mathrm{N}_{2} \mathrm{O}$ emissions. Hickman et al. [40] showed that plots treated with $200 \mathrm{~kg} \mathrm{Nha}^{-1}$ registered $24 \%$ more $\mathrm{N}_{2} \mathrm{O}$ emissions than plots that received no fertilizer. In a maize-based study by Nyamadzawo et al. [53] in Zimbabwe, the use of inorganic fertilizers emitted 0.35 to $0.52 \mathrm{~kg} \mathrm{~N}_{2} \mathrm{O}-\mathrm{N} \mathrm{ha}^{-1}$ compared to control that emitted $0.32 \mathrm{~kg} \mathrm{~N}_{2} \mathrm{O}-\mathrm{N} \mathrm{ha}{ }^{-1}$. Ortiz-Gonzalo et al. [43] reported $65 \%$ of total $\mathrm{N}_{2} \mathrm{O}$ emissions in fertilized plots than unfertilized plots under cereal-legume and coffee cropping systems in Kenya. For instance, a higher fertilizer rate of more than $100 \mathrm{~kg} \mathrm{~N} \mathrm{ha}^{-1} \mathrm{yr}^{-1}$ emitted 3.49 to $4.29 \mathrm{~kg} \mathrm{~N}_{2} \mathrm{O}-\mathrm{N} \mathrm{ha}^{-1}$. In comparison, fertilizer rates below $100 \mathrm{~kg} \mathrm{Nha}^{-1} \mathrm{yr}^{-1}$ recorded 1.22 and $1.79 \mathrm{~kg} \mathrm{~N}_{2} \mathrm{O}-\mathrm{N}$ $\mathrm{ha}^{-1}$ in Ghana under maize cropping systems [45], while in Iringa and Mbeya sites in Tanzania, inorganic fertilizer plots planted with maize crops emitted between 0.14 to $0.44 \mathrm{~kg}$ and 0.18 to $0.72 \mathrm{~kg} \mathrm{~N}_{2} \mathrm{O}-\mathrm{N} \mathrm{ha}^{-1}$, respectively [46]. Further, from Macharia et al. [28], inorganic fertilized plots under maize cropping systems emitted $10 \%$ more $\mathrm{N}_{2} \mathrm{O}$ emissions than control plots in the Central Highlands of Kenya. In comparison, Musafiri et al. [48] in the same region reported $98 \%$ more $\mathrm{N}_{2} \mathrm{O}$ emissions from inorganic fertilizer treatment than the control treatment. The above studies show that an increase in $\mathrm{N}$ application results in higher $\mathrm{N}_{2} \mathrm{O}$ emissions regardless of fertilizer type and cropping system.

Nevertheless, contrasting results on $\mathrm{N}_{2} \mathrm{O}$ emissions in SSA on the use of organic and inorganic inputs have been reported. Dick et al. [35] reported less $\mathrm{N}_{2} \mathrm{O}$ emissions in plots treated with both organic and inorganic fertilizer $\left(0.9 \mathrm{~kg} \mathrm{~N}_{2} \mathrm{O}-\mathrm{N} \mathrm{ha}^{-1}\right)$ than plots treated with sole manure $\left(1.5 \mathrm{~kg} \mathrm{~N}_{2} \mathrm{O}-\mathrm{N} \mathrm{ha}^{-1}\right)$ in semiarid areas of Mali. Similarly, increasing $\mathrm{N}$ input through the combination of inorganic fertilizer $\left(60 \mathrm{~kg} \mathrm{~N}_{2} \mathrm{O}-\mathrm{N} \mathrm{ha}^{-1}\right)$ and manure $\left(97.5 \mathrm{~kg} \mathrm{~N} \mathrm{ha}^{-1}\right)$ increases $\mathrm{N}_{2} \mathrm{O}$ emissions by 22 times more than control plots under rape fruits in Zimbabwe [39]. Positive $\mathrm{N}$ balance was reported in the combination of organic and inorganic fertilizer during maize growth in western Kenya compared with sole inorganic fertilizer application [71]. However, Nyamadzawo et al. [53] found that sole manure application reduces $\mathrm{N}_{2} \mathrm{O}$ emissions by $16 \%$ compared to sole inorganic and integrated application, which increases $\mathrm{N}_{2} \mathrm{O}$ emissions by 28 and $9 \%$, respectively in reference to control in Zimbabwe. The combination of inorganic and organic fertilizer as reported by [43] in two farms were 3- and 5-fold higher than unfertilized maize and coffee cropping systems, respectively, in Thara farm while in Kahau farm, fertilized plot registered 2- and 6-fold higher $\mathrm{N}_{2} \mathrm{O}$ emissions than unfertilized plots for coffee and maize plots, respectively. The combination of inorganic fertilizer and maize stover treatments (150 kg N ha ${ }^{-1}$ each) had significantly higher $\mathrm{N}_{2} \mathrm{O}$ emissions ( 0.55 to $2.2 \mathrm{~kg} \mathrm{Nha}^{-1}$ ) compared to sole fertilizer application at the same rate $(0.34$ to $0.72 \mathrm{~kg} \mathrm{Nha}^{-1}$ ) in Tanzania [46]. A combination of organic and inorganic manure increased $\mathrm{N}_{2} \mathrm{O}$ emissions five-fold compared to control under maize cropping systems in Central Highland Kenya [21]. Additionally, Musafiri et al. [48] reported 1.5 times more $\mathrm{N}_{2} \mathrm{O}$ in inorganic plots than in control. The above studies found that a combination of organic and inorganic fertilizer provides mixed results concerning $\mathrm{N}_{2} \mathrm{O}$ emissions. It is worth noting that a combination of organic and inorganic fertilizers can only lower $\mathrm{N}_{2} \mathrm{O}$ emissions when organic manure with a low $\mathrm{C} / \mathrm{N}$ ratio and inorganic fertilizer with high $\mathrm{N}$ levels are combined.

\section{Yield-Scaled $\mathrm{N}_{2} \mathrm{O}$ Emissions (YSE) and Nitrous Oxide Emissions Factors (EFs)}

4.1. Yield-Scaled $\mathrm{N}_{2} \mathrm{O}$ Emissions (YSE). Farm management activities for cropping systems should improve soil fertility, agronomic productivity, and environmental sustainability. Yield-scaled emissions (YSE) relate to $\mathrm{N}_{2} \mathrm{O}$ emissions and crop yields expressed as emissions per unit yield that can be used to assess management impact [72]. The amount of $\mathrm{N}_{2} \mathrm{O}$ emitted determines the amount of YSE. Therefore, the YSE parameter provides an entry point to evaluate the ability of management to mitigate $\mathrm{N}_{2} \mathrm{O}$ emissions without compromising productivity [72]. Limited studies have attempted to determine YSE on different cropping systems based on inorganic and organic fertilizer applications in SSA. The YSE reported in a few studies ranges between 0.02 and $67.7 \mathrm{~g} \mathrm{~N}_{2} \mathrm{O}-\mathrm{N} \mathrm{kg}^{-1}$. For instance, Mapanda et al. [52] reported YSE emissions ranging from 0.02 to $3.93 \mathrm{~g} \mathrm{~N} 2 \mathrm{O}-\mathrm{N}$ $\mathrm{kg}-1$ in Zimbabwe under maize cropping systems. Nyamadzawo et al. [53] reported YSE of $0.26 \mathrm{~g} \mathrm{~N}_{2} \mathrm{O}-\mathrm{N} \mathrm{kg}^{-1}$ yield from integrated fertilizer management for maize cropping systems in Zimbabwe. Pelster et al. [1] reported YSE between 1.1 and $67 \mathrm{~g} \mathrm{~N}_{2} \mathrm{O}-\mathrm{N} \mathrm{kg} \mathrm{kg}^{-1}$ aboveground uptake in maize cropping systems in Eastern Africa. Findings from a maize cropping system in Ghana by Atakora et al. [45] showed that $\mathrm{N}$ fertilization above $100 \mathrm{~kg} \mathrm{Nha}^{-1} \mathrm{yr}^{-1}$ led to $1.24 \mathrm{~g} \mathrm{~N}_{2} \mathrm{O} \mathrm{kg}^{-1}$ grain, while $\mathrm{N}$ fertilization below $100 \mathrm{~kg} \mathrm{ha}^{-}$ ${ }^{1} \mathrm{yr}^{-1}$ resulted in less than $0.6 \mathrm{~g} \mathrm{~N}_{2} \mathrm{O} \mathrm{kg}^{-1}$ grain. In the central highlands of Kenya, Macharia et al. [28] reported YSE ranging from 0.5 to $2.2 \mathrm{~g} \mathrm{~N}_{2} \mathrm{O}-\mathrm{N} \mathrm{kg-}{ }^{1}$ grain yield under manure and a combination of manure and inorganic fertilizer, respectively. Similarly, Musafiri et al. [48] reported YSE ranging from 0.024 to $0.028 \mathrm{~g} \mathrm{~N}_{2} \mathrm{O}-\mathrm{N} \mathrm{kg}^{-1}$ grain yield from sole manure and inorganic fertilizer, respectively, in Central Highland of Kenya. Yield-scaled emissions reported in most SSA farming systems may be associated with existing climate variability and soil fertility decline, which could have lowered crop yield, which determines YSE other than higher $\mathrm{N}_{2} \mathrm{O}$ emissions. 
4.2. Nitrous Oxide Emissions Factors (EFs). Limited studies in SSA notwithstanding, most of the derived EFs were below $1 \%$ and ranged between 0.1 and $0.9 \%$ across cropping systems in SSA [9, 28, 41, 46, 48, 49, 57]. However, there were a few instances where EFs exceeded $1 \%$ from maize, vegetable cropping systems, and soil laboratory incubation studies [35, 37]. In Mali, for instance, Dick et al. [35] reported the highest EFs for maize cropping of $4.1 \%$, attributed to field management interferences where higher fluxes of $\mathrm{N}_{2} \mathrm{O}$ were noted even before applying fertilizer. For maize cropping systems in Kenya, $\mathrm{N}_{2} \mathrm{O}$ EFs ranged from 0.01 to 0.9\% (e.g., Baggs et al. [18], Hickman et al. [41], Macharia et al., [28] and Musafiri et al. [48]) while in Zimbabwe, it was below $0.2 \%$ [49]. Similarly, in Tanzania, EFs ranged between 0.13 and $0.42 \%$ [46]. Rosenstock et al. [42] showed that tea cropping EFs were below $1 \%$ in Kenya. The $\mathrm{N}_{2} \mathrm{O}$ emission factors from vegetable cropping systems in Zimbabwe ranged from 0.3 to $4 \%$ and were attributed to high fertilizer application $[37,70]$. The EFs mentioned above are largely below IPCC tier 1 defaults, suggesting that default EFs on SSA's GHG emissions estimations may overestimate it, resulting in incorrect targeting of adaptation and mitigation measures. These findings may also show that "umbrella" recommendation of adaptation and mitigation measures may not accurately be applicable in a different place with different climatic, environmental, and farm management practices.

\section{Drivers of Soil $\mathrm{N}_{2} \mathrm{O}$ Emissions in SSA}

Studies across SSA have documented varied soil $\mathrm{N}_{2} \mathrm{O}$ emissions under different environmental, climatic, and soil conditions and farm management practices $[1,9,28,45,53,73]$. Environmental factors (land use land cover changes), soil properties (bulk density, temperature, moisture, $\mathrm{pH}$, type, organic carbon, and nitrogen), and climatic factors (temperature and precipitation) may significantly influence soil $\mathrm{N}_{2} \mathrm{O}$ fluxes. Similarly, farm management practices, including fertilizer application (rates, time, type, and method), tillage, crop type, and residue management, may also influence $\mathrm{N}_{2} \mathrm{O}$ emissions. It is noteworthy that these factors do not occur singly, but their interactive effects determine whether the soil is a net sink or source of $\mathrm{N}_{2} \mathrm{O}$ emissions [42].

\subsection{Effects of Soil Temperature and Elevation on $\mathrm{N}_{2} \mathrm{O}$ Emis-} sions in SSA. Soil temperature significantly influences soil $\mathrm{N}_{2} \mathrm{O}$ fluxes by increasing microbial activities responsible for $\mathrm{N}_{2} \mathrm{O}$ emissions in soil [27]. Nitrous oxide emissions increase with an increase in soil temperature due to advanced decomposition rates of organic matter [58]. An increase in $\mathrm{N}_{2} \mathrm{O}$ emissions with rising temperature can be associated with increased nitrogen mineralization, hence higher availability of nitrogen lost as $\mathrm{N}_{2} \mathrm{O}$ fluxes [27]. However, denitrifying bacteria are susceptible to soil temperature and operate best at an optimum temperature of $30^{\circ} \mathrm{C}$, beyond which activities go down, thus lowering $\mathrm{N}_{2} \mathrm{O}$ emissions [74]. Various studies in SSA, such as Mapanda et al. [52] in
Zimbabwe, Lompo et al. [37] in Burkina Faso, Rosenstock et al. [42] in Kenya, and Atakora et al. [45] in Ghana, have reported a significant positive correlation between soil temperature and nitrous oxide emissions.

Atmospheric temperature also influences $\mathrm{N}_{2} \mathrm{O}$ emissions. For instance, Fatumah et al. [58] reported higher $\mathrm{N}_{2} \mathrm{O}$ emissions in higher altitudes (1200-1300 m above sea level (asl)) characterized with low temperatures as compared to low altitudes (1100 to $1200 \mathrm{~m}$ and 900 to $1100 \mathrm{~m}$ asl) with higher temperature in Uganda. Further, in Kenya, higher soil $\mathrm{N}_{2} \mathrm{O}$ emissions were observed in Kaptumo with an elevation of $2000 \mathrm{~m}$ asl than Kolero with an elevation of $1250 \mathrm{~m}$ asl [42]. Notably, higher elevations recorded greater soil $\mathrm{C}$ and $\mathrm{N}$, resulting in high $\mathrm{N}_{2} \mathrm{O}$ fluxes. However, atmospheric temperature decreases with increasing elevation; therefore, it may influence soil microbial activities responsible for $\mathrm{N}_{2} \mathrm{O}$ emissions.

\subsection{Effects of Soil Moisture Content on $\mathrm{N}_{2} \mathrm{O}$ Emissions in SSA.} Soil moisture is a crucial driver of $\mathrm{N}_{2} \mathrm{O}$ fluxes as it determines oxygen and organic substrates' availability [24]. Several studies have shown that increased soil moisture content, especially at the onset of a season, results in increased microbial activities causing enhanced soil $\mathrm{N}_{2} \mathrm{O}$ emissions [28, 43, 44, 58, 64]. Increased $\mathrm{N}_{2} \mathrm{O}$ emissions following the beginning of precipitation can be attributed to improved soil CN mineralization and decomposition due to Birch's birch effect [75]. Sufficient anaerobic microsites for the denitrification process increase $\mathrm{N}_{2} \mathrm{O}$ emissions [12]. It also increases bacterial growth and other activities, thereby increasing respiration rates and soil aeration. Soil moisture increases nutrients' transport to soil microbes responsible for the denitrification process. For instance, in a study by Rabenarivo et al. [76] in Madagascar, an increase in soil moisture from $40 \%$ to $90 \%$ amplified $\mathrm{N}_{2} \mathrm{O}$ fluxes by about $46 \%$. In Kenya, Macharia et al. [28] observed that a difference of $32 \%$ in rainfall amounts between long rains and short rains seasons of 2017 resulted in a variance of four to six times more $\mathrm{N}_{2} \mathrm{O}$ emissions across treatments. Soil moisture influences $\mathrm{N}_{2} \mathrm{O}$ emissions by activating microbial activities that lead to denitrification and nitrification processes.

5.3. Effects of Soil Properties on $\mathrm{N}_{2} \mathrm{O}$ Emissions in SSA. Soil physicochemical properties play a crucial role in $\mathrm{N}_{2} \mathrm{O}$ dynamics by controlling soil carbon and nitrogen availability [77]. Soil texture influences water holding capacity and gas diffusivity rate and, therefore, regulates oxygen availability, enhancing microbial activities [12]. Course soil texture emits less $\mathrm{N}_{2} \mathrm{O}$ emissions than fine texture due to the high accumulation of oxygen that limits denitrification rates, which is an ideal process for $\mathrm{N}_{2} \mathrm{O}$ emissions production [78]. On the other hand, clayey soil microsites pores contribute to $\mathrm{N}_{2} \mathrm{O}$ emissions production by enhancing anaerobic conditions favorable for denitrification. Studies have documented that adding inorganic fertilizers on fine-textured soil and organic fertilizer on coarse-textured soil significantly increases $\mathrm{N}_{2} \mathrm{O}$ oxide emissions [28, 52, 53]. Concerning soil 
type, coarse-textured soils are generally $\mathrm{C}$ deficient, while fine-textured soil is generally $\mathrm{N}$ deficient [79]. The application of organic fertilizer on coarse-textured soil supplies mineralizable $\mathrm{C}$ hence stimulating $\mathrm{N}_{2} \mathrm{O}$ emissions in carbonlimited soils, while applying inorganic fertilizer in fine soil supply $\mathrm{N}$, providing substrate for microbial community, thus increasing $\mathrm{N}_{2} \mathrm{O}$ emissions.

Soil bulk density influences $\mathrm{N}_{2} \mathrm{O}$ fluxes by regulating oxygen diffusion into soils, which is essential for nitrification [12]. It limits soil aeration, which enhances the production of $\mathrm{N}_{2}$ into the atmosphere through diffusion. Wanyama et al. [44] found a negative correlation between bulk density and soil $\mathrm{N}_{2} \mathrm{O}$ fluxes, implying that increased bulk density and higher soil compaction result in lower $\mathrm{N}_{2} \mathrm{O}$ emissions.

Soil pH significantly affects $\mathrm{N}_{2} \mathrm{O}$ emissions as it controls bacterial activities, nutrient availability, and soil structure. Nitrification-denitrification microbes are $\mathrm{pH}$-sensitive; hence, their alteration determines $\mathrm{N}_{2} \mathrm{O}$ emissions. Low soil $\mathrm{pH}$ may alter functions of $\mathrm{N}_{2} \mathrm{O}$ reductase enzymes, which are responsible for reducing $\mathrm{N}_{2} \mathrm{O} / \mathrm{N}_{2}$ ratio and, therefore, may lead to higher $\mathrm{N}_{2} \mathrm{O}$ emissions [80]. Under acidic soils, an increase in soil $\mathrm{pH}$ leads to less $\mathrm{N}_{2} \mathrm{O}$ emissions though $\mathrm{N}_{2} \mathrm{O}$ emissions increase with a decrease in $\mathrm{pH}$ under alkaline soil [81]. The manure application may also contribute to an increase in soil $\mathrm{pH}$, resulting in lower $\mathrm{N}_{2} \mathrm{O}$ emissions [43]. However, Macharia et al. [28] found higher $\mathrm{N}_{2} \mathrm{O}$ emissions in manure-treated plots despite having the highest $\mathrm{pH}$. Consequently, caution should be taken during continuous fertilizer application since it may promote soil acidification and encourage $\mathrm{N}_{2} \mathrm{O}$ emissions.

Soil $\mathrm{N}_{2} \mathrm{O}$ emissions can significantly be influenced by soil $\mathrm{C}, \mathrm{N}$, and $\mathrm{C} / \mathrm{N}$ ratio [58]. The $\mathrm{C} / \mathrm{N}$ ratio can predict whether mineralization or immobilization takes place. Use of crop residue with a high $\mathrm{C} / \mathrm{N}$ ratio results in prolonged decomposition of organic matter. Consequently, a combination of low-quality crop residue with high-quality manure may offset $\mathrm{N}$ loss by balancing $\mathrm{C} / \mathrm{N}$, therefore, reducing immobilization. Low soil organic carbon limits denitrification and microbial activity resulting in lower $\mathrm{N}_{2} \mathrm{O}$ emissions [42]. Studies have reported a positive correlation between inorganic nitrogen $\left(\mathrm{NO}_{3}-\mathrm{N}, \mathrm{NH}_{4}{ }^{+}-\mathrm{N}\right.$, and IN) and $\mathrm{N}_{2} \mathrm{O}$ fluxes $[1,28,44,50]$. Both soil $\mathrm{C}$ and $\mathrm{N}$ influence $\mathrm{N}_{2} \mathrm{O}$ fluxes; therefore, the soil $\mathrm{C} / \mathrm{N}$ ratio is an essential predictor of $\mathrm{N}_{2} \mathrm{O}$ emissions. For instance, Wanyama et al. [44] documented a negative correlation between soil $\mathrm{C} / \mathrm{N}$ ratio and $\mathrm{N}_{2} \mathrm{O}$ fluxes which was attributed to a potential decrease in $\mathrm{N}$ mineralization with an increase in $\mathrm{C}: \mathrm{N}$ ratio.

\section{Soil $\mathrm{N}_{2} \mathrm{O}$ Emissions' Mitigation Options in SSA}

Given the diversity of soil $\mathrm{N}_{2} \mathrm{O}$ fluxes drivers in SSA, no single mitigation option is applicable across all agroecological conditions. Therefore, a targeted approach specific to an agroecological zone is necessary for recommending different SSA interventions. For instance, soil type-soil fertility management targeting is an appropriate mechanism in evaluating $\mathrm{N}_{2} \mathrm{O}$ emissions mitigation. Organic manure, which is readily available in most SSA households (e.g., Macharia et al. [67] and Nganga et al. [68]) may provide an essential entry point in mitigating $\mathrm{N}_{2} \mathrm{O}$ emissions in SSA. Manure enriches soils with mineralizable $\mathrm{C}$ and $\mathrm{N}$, thus improving soil fertility, general soil health, and crop yields but may come at the cost of more $\mathrm{N}_{2} \mathrm{O}$ emissions [21]. However, with most of the landmass in SSA being arid and semiarid (ASALs) (45-55\%), there is a need to achieve a nexus between crop production and $\mathrm{N}_{2} \mathrm{O}$ emissions [82]. Therefore, $\mathrm{N}_{2} \mathrm{O}$ yield-scaled emissions, which compare $\mathrm{N}_{2} \mathrm{O}$ emissions and crop yields, can be used to assess the suitability of effective mitigation options.

\subsection{Integrated Soil Fertility Management (ISFM).}

Integrated soil fertility management is an agricultural practice that combines locally available organic resources, improved germplasm, and mineral fertilizers to enhance nutrients and water efficiency to increase crop production $[66,83]$. Combining manure and inorganic fertilizers has increased agricultural productivity while mitigating $\mathrm{N}_{2} \mathrm{O}$ emissions in sandy loam (moderate texture) soils in Zimbabwe by increasing mineralizable C [53]. Sommer et al. [71] also found that integrated soil fertility management practices can improve $\mathrm{N}$ balance and contribute to environmental sustainability better than either sole inorganic fertilizer or organic fertilizer application. Crop residue retention can increase agricultural productivity with lower $\mathrm{N}_{2} \mathrm{O}$ emissions compared with inorganic fertilizers [84]. Integrated soil fertility management contributes to the mitigation of $\mathrm{N}_{2} \mathrm{O}$ emissions by improving soil health and crop productivity, increasing yield, and reducing YSEs.

6.2. Cereal-Legume Intercropping. Cereal-legume intercropping enhances soil and crop productivity through nitrogen fixation and soil conservation. Leguminous crops have a low $\mathrm{C} / \mathrm{N}$ ratio than cereal crops [85]. This implies that combining both cereals and legumes in the field may reduce N's immobilization in soil and increase the availability of $\mathrm{N}$ and better synchronization by plant. Therefore, cereal-legume intercrop/rotation targeting might be an essential entry point in mitigating soil $\mathrm{N}_{2} \mathrm{O}$ fluxes among smallholder farming systems in SSA. For instance, cereal-legume intercropping or rotation might enhance soil $\mathrm{N}_{2} \mathrm{O}$ mitigation. Dick et al. [35] found significantly low soil $\mathrm{N}_{2} \mathrm{O}$ fluxes under cereal-legume rotation cropping in Mali. According to Frimpong et al. [73], cowpea-maize intercrop emitted lower $\mathrm{N}_{2} \mathrm{O}$ emissions than cowpea alone. The use of cereallegume rotation improves nitrogen fixation, thus reducing the need for inorganic fertilizer [86], which, if applied, could lead to more $\mathrm{N}_{2} \mathrm{O}$ fluxes. The cereal-legume in SSA can fix approximately 15 to $210 \mathrm{~kg} \mathrm{Nha}^{-1}$ [87], thus improving soil fertility. This implies that the use of legumes, intercropped, or rotation may reduce $\mathrm{N}$ mining of maize crops currently ranging between 14 and $110 \mathrm{~kg} \mathrm{Nha}^{-1} \mathrm{yr}^{-1}$ in SSA [88]. In addition to reducing $\mathrm{N}_{2} \mathrm{O}$ emissions and improving soil fertility, farmers practicing cereal-legume intercropping spread risk of crop failure in climate variability, hence increasing their economic plausibility and nutritional security [89]. 
6.3. Fertilizer Application Management. Fertilizer application management is very crucial for plant growth and $\mathrm{N}$ cycle in soil. Effective $\mathrm{N}$ fertilizer management in farm needs to consider N's amount required by plants and $\mathrm{N}$ supplied. This is because $\mathrm{N}$ application to soils might significantly influence $\mathrm{N}_{2} \mathrm{O}$ emissions in SSA [41]. For instance, even though Hickman et al. [40] observed no significant difference in $\mathrm{N}_{2} \mathrm{O}$ fluxes between fertilizer application rates in Western Kenya, emissions increased with an increased fertilizer application rate. This implies that applying the right amount of $\mathrm{N}$ to soil could significantly mitigate $\mathrm{N}_{2} \mathrm{O}$ emissions instead of countries' specific blanket fertilizer application recommendations.

Further, the determination of site-specific fertilizer type can be essential in mitigating $\mathrm{N}_{2} \mathrm{O}$ emissions. The use of nitrogen inhibitors and split application can also be necessary for reducing $\mathrm{N}_{2} \mathrm{O}$ emissions from smallholder cropping systems in SSA. Finally, Nafi et al. [90] documented that microdosing (fertilizer application at the root) lowered $\mathrm{N}$ losses. Therefore, establishing site-specific $4 \mathrm{Rs}$ of fertilizer application (right time, right rate, right place, and right type) is requisite for mitigating $\mathrm{N}_{2} \mathrm{O}$ emissions in SSA.

6.4. Reduce/No-Tillage Option. Soil disturbance through tillage could significantly increase $\mathrm{N}_{2} \mathrm{O}$ emissions by altering soil physical properties such as bulk density. Tillage method targeting can offer bases for $\mathrm{N}_{2} \mathrm{O}$ fluxes mitigation among smallholder cropping systems in SSA. For instance, Chikowo et al. [49] and Baggs et al. [18] documented lower $\mathrm{N}_{2} \mathrm{O}$ emissions under no-tillage than tilled farms. Since conservation tillage (no-tillage or minimum tillage) increases agricultural productivity and lowers $\mathrm{N}_{2} \mathrm{O}$ emissions, their adoption among smallholder farmers in SSA could mitigate the effects of $\mathrm{N}_{2} \mathrm{O}$ emissions.

\section{Conclusion}

A better understanding of soil $\mathrm{N}_{2} \mathrm{O}$ emissions, YSE, and EFs from different cropping systems in SSA is essential in promoting agricultural sustainability and climate change mitigation. The finding from the SSA studies agrees with our hypothesis that $\mathrm{N} 2 \mathrm{O}$ emissions significantly differ across cropping systems. However, $\mathrm{N}_{2} \mathrm{O}$ emissions remained relatively low compared to global averages, except for vegetable cropping systems mainly due to inherently low soil fertility due to continuous farming with limited replenishment with external inputs. We found out that better nutrient management through the combination of organic and inorganic fertilizers could provide a viable option in mitigating $\mathrm{N}_{2} \mathrm{O}$ emissions in SSA. Our review also reveals that SSA's EFs are lower than IPCC Tier 1 default EFs meaning that the use of default EFs may overestimate soil $\mathrm{N}_{2} \mathrm{O}$ emissions and lead to inaccurate targeting of climate change adaptation and mitigation measures in SSA. However, a few exceptional cases, mainly from vegetable production and applied more fertilizers comparatively, documented more than $1 \%$ in SSA. Our review identified environmental, climatic, and soil properties as critical drivers that significantly influence $\mathrm{N}_{2} \mathrm{O}$ fluxes dynamics in SSA. Our study revealed that "umbrella" (universal) recommendations for climate change mitigation measures might not be effective across SSA cropping systems based on their diversity. Therefore, devising site-specific mitigation interventions could be a plausible entry point to mitigate $\mathrm{N}_{2} \mathrm{O}$ emissions. We singled out options for targeting $\mathrm{N}_{2} \mathrm{O}$ emissions mitigation in SSA: integrated soil fertility management; cereal-legume intercropping; reduced/ no-tillage; and improved fertilizer application management. We recommend establishing more empirical studies in area with varying agro-ecological zones and soil types in SSA to qualify various mitigation options on $\mathrm{N}_{2} \mathrm{O}$ emissions, yieldscaled $\mathrm{N}_{2} \mathrm{O}$ emissions, and $\mathrm{N}_{2} \mathrm{O}$ emission factors, which are essential in improving national and regional GHG inventories.

\section{Data Availability}

The data supporting the findings of the study are available upon request from the corresponding author.

\section{Conflicts of Interest}

The authors declare no conflicts of interest.

\section{Acknowledgments}

The authors acknowledge Kenya National Research Fund for the financial grant through the University of Embu titled

"Towards Quantifying Green House Gas Emissions and Deriving Emission Factors from Organic and Inorganic Fertilized Farming Systems of Kenya."

\section{References}

[1] D. Pelster, M. Rufino, T. Rosenstock et al., "Smallholder farms in eastern African tropical highlands have low soil greenhouse gas fluxes," Biogeosciences, vol. 14, no. 1, pp. 187-202, 2017.

[2] IPCC, "Climate change 2014: synthesis report," in Contribution of Working Groups I, II and III to the Fifth Assessment Report of the Intergovernmental Panel on Climate Change, R. K. Pachauri and L. A. Meyer, Eds., IPCC, Geneva, Switzerland, 2014.

[3] I. S. Thakur and K. Medhi, "Nitrification and denitrification processes for mitigation of nitrous oxide from waste water treatment plants for biovalorization: challenges and opportunities," Bioresource Technology, vol. 282, pp. 502-513, 2019.

[4] N. N. Omoyo, J. Wakhungu, and S. Oteng'i, "Effects of climate variability on maize yield in the arid and semi-arid lands of lower eastern Kenya," Agriculture \& Food Security, vol. 4, pp. 1-13, 2015.

[5] M. Kabirigi, B. Musana, F. K. Ngetich, and J. Mugwe, "Applicability of conservation agriculture for climate change adaptation in Rwanda's situation," Journal of Soil Science and Environmental Management, vol. 6, pp. 241-248, 2015.

[6] A. R. Ravishankara, J. S. Daniel, and R. W. Portmann, "Nitrous oxide $\left(\mathrm{N}_{2} \mathrm{O}\right)$ : the dominant ozone-depleting substance emitted in the 21st century," Science, vol. 326, no. 5949, pp. 123-125, 2009.

[7] R. Barnard, P. W. Leadley, and B. A. Hungate, "Global change, nitrification, and denitrification: a review," Global Biogeochemical Cycles, vol. 19, pp. 1-13, 2005. 
[8] W. Eugster and L. Merbold, "Eddy covariance for quantifying trace gas fluxes from soils," Soils, vol. 1, no. 1, pp. 187-205, 2015.

[9] E. M. Baggs, J. Chebii, and J. K. Ndufa, "A short-term investigation of trace gas emissions following tillage and notillage of agroforestry residues in western Kenya," Soil and Tillage Research, vol. 90, no. 1-2, pp. 69-76, 2006.

[10] M. A. Altieri and P. Koohafkan, Enduring Farms: Climate Change, Smallholders and Traditional Farming Communities, Vol. 6, Third World Network (TWN), Penang, Malaysia, 2008.

[11] F. Beeckman, H. Motte, and T. Beeckman, "Nitrification in agricultural soils: impact, actors and mitigation," Current Opinion in Biotechnology, vol. 50, pp. 166-173, 2018.

[12] K. Butterbach-Bahl, E. M. Baggs, M. Dannenmann, R. Kiese, and S. Zechmeister-Boltenstern, "Nitrous oxide emissions from soils: how well do we understand the processes and their controls?" Philosophical Transactions of the Royal Society B: Biological Sciences, vol. 368, no. 1621, Article ID 20130122, 2013.

[13] C. Hénault, A. Grossel, B. Mary, M. Roussel, and J. Léonard, "Nitrous oxide emission by agricultural soils: a review of spatial and temporal variability for mitigation," Pedosphere, vol. 22, no. 4, pp. 426-433, 2012.

[14] P. Ciais, C. Sabine, and G. Bala, "Carbon and other biogeochemical cycles," in Climate Change 2013: The Physical Science Basis. Contribution of Working Group I to the Fifth Assessment Report of the Intergovernmental Panel on Climate Change, pp. 465-570, Cambridge University Press, Cambridge, UK, 2014.

[15] I. Vermeulen, C. Block, and C. Vandecasteele, "Estimation of fuel-nitrogen oxide emissions from the element composition of the solid or waste fuel," Fuel, vol. 94, pp. 75-80, 2012.

[16] S. K. Jalota, B. B. Vashisht, S. Sharma, and S. Kaur, Understanding Climate Change Impacts on Crop Productivity and Water Balance, Academic Press, Cambridge, MA, USA, 2018.

[17] P. Tittonell, B. Vanlauwe, P. A. Leffelaar, E. C. Rowe, and K. E. Giller, "Exploring diversity in soil fertility management of smallholder farms in western Kenya," Agriculture, Ecosystems \& Environment, vol. 110, no. 3-4, pp. 149-165, 2005.

[18] E. M. Baggs, "Soil microbial sources of nitrous oxide: recent advances in knowledge, emerging challenges and future direction," Current Opinion in Environmental Sustainability, vol. 3, no. 5, pp. 321-327, 2011.

[19] J. E. Hickman, M. Havlikova, C. Kroeze, and C. A. Palm, "Current and future nitrous oxide emissions from African agriculture," Current Opinion in Environmental Sustainability, vol. 3, no. 5, pp. 370-378, 2011.

[20] D.-G. Kim, A. D. Thomas, D. Pelster, T. S. Rosenstock, and A. Sanz-Cobena, "Greenhouse gas emissions from natural ecosystems and agricultural lands in sub-Saharan Africa: synthesis of available data and suggestions for further research," Biogeosciences, vol. 13, no. 16, pp. 4789-4809, 2016.

[21] M. I. Tongwane and M. E. Moeletsi, "A review of greenhouse gas emissions from the agriculture sector in Africa," Agricultural Systems, vol. 166, pp. 124-134, 2018.

[22] D. Ortiz-Gonzalo, P. Vaast, M. Oelofse, A. de Neergaard, A. Albrecht, and T. S. Rosenstock, "Farm-scale greenhouse gas balances, hotspots and uncertainties in smallholder croplivestock systems in Central Kenya," Agriculture, Ecosystems \& Environment, vol. 248, pp. 58-70, 2017.

[23] C. M. Musafiri, J. M. Macharia, O. K. Ng'etich et al., "Farming systems' typologies analysis to inform agricultural greenhouse gas emissions potential from smallholder rain-fed farms in Kenya," Scientific African, vol. 8, Article ID e00458, 2020.

[24] K. A. Frimpong, D. O. Yawson, K. Agyarko, and E. M. Baggs, " $\mathrm{N}_{2} \mathrm{O}$ emission and mineral $\mathrm{N}$ release in a tropical acrisol incorporated with mixed cowpea and maize residues," Agronomy, vol. 2, no. 3, pp. 167-186, 2012.

[25] S. S. Snapp, C. M. Cox, and B. G. Peter, "Multipurpose legumes for smallholders in Sub-Saharan Africa: identification of promising "scale out" options," Global Food Security, vol. 23, pp. 22-32, 2019.

[26] M. C. Rufino, P. Brandt, M. Herrero, and K. Butterbach-Bahl, "Reducing uncertainty in nitrogen budgets for African livestock systems," Environmental Research Letters, vol. 9, no. 10, Article ID 105008, 2014.

[27] P. K. Thornton and M. Herrero, "Adapting to climate change in the mixed crop and livestock farming systems in SubSaharan Africa," Nature Climate Change, vol. 5, no. 9, pp. 830-836, 2015.

[28] M. J. Macharia, E. D. Pelster, and F. K. Ngetich, "Soil greenhouse gas fluxes from maize production under different soil fertility management practices in East Africa," Journal of Geophysical Research: Biogeoscience, vol. 125, 2020.

[29] V. Gyanchandani, "UNFCCC nationally determined contributions: climate change and trade," 2016.

[30] R. Elkahwagy, V. Gyanchandani, and D. Piselli, UNFCCC Nationally Determined Contributions: Climate Change and Trade, Center for Trade and Economic Integration (CTEI) Working Paper, 2017.

[31] UNFCCC, Adoption of the Paris Agreement. I: Proposal by the President (Draft Decision), United Nations Office, Geneva, Switzerland, 2015.

[32] M. Richards, R. Metzel, N. Chirinda et al., "Limits of agricultural greenhouse gas calculators to predict soil $\mathrm{N}_{2} \mathrm{O}$ and $\mathrm{CH}_{4}$ fluxes in tropical agriculture," Scientific Reports, vol. 6, pp. 26279-26288, 2016.

[33] F. Albanito, U. Lebender, T. Cornulier et al., "Direct nitrous oxide emissions from tropical and sub-tropical agricultural systems-a review and modelling of emission factors," Scientific Reports, vol. 7, pp. 44235-44312, 2017.

[34] C. Brümmer, H. Papen, R. Wassmann, and N. Brüggemann, "Termite mounds as hot spots of nitrous oxide emissions in South-Sudanian savanna of Burkina Faso (West Africa)," Geophysical Research Letters, vol. 36, pp. 1-4, 2009.

[35] J. Dick, B. Kaya, M. Soutoura et al., "The contribution of agricultural practices to nitrous oxide emissions in semi-arid Mali," Soil Use \& Management, vol. 24, no. 3, pp. 292-301, 2008.

[36] M. Predotova, J. Gebauer, R. V. C. Diogo, E. Schlecht, and A. Buerkert, "Emissions of ammonia, nitrous oxide and carbon dioxide from urban gardens in Niamey, Niger," Field Crops Research, vol. 115, no. 1, pp. 1-8, 2010.

[37] D. J.-P. Lompo, S. A. K. Sangaré, E. Compaoré et al., “Gaseous emissions of nitrogen and carbon from urban vegetable gardens in Bobo-Dioulasso, Burkina Faso," Journal of Plant Nutrition and Soil Science, vol. 175, no. 6, pp. 846-853, 2012.

[38] F. Mapanda, M. Wuta, J. Nyamangara, and R. M. Rees, "Nitrogen leaching and indirect nitrous oxide emissions from fertilized croplands in Zimbabwe," Nutrient Cycling in Agroecosystems, vol. 94, no. 1, pp. 85-96, 2012.

[39] G. Nyamadzawo, M. Wuta, J. Nyamangara, J. L. Smith, and R. M. Rees, "Nitrous oxide and methane emissions from cultivated seasonal wetland (dambo) soils with inorganic, organic and integrated nutrient management," Nutrient Cycling in Agroecosystems, vol. 100, no. 2, pp. 161-175, 2014. 
[40] J. E. Hickman, C. A. Palm, P. Mutuo, J. M. Melillo, and J. Tang, "Nitrous oxide $\left(\mathrm{N}_{2} \mathrm{O}\right)$ emissions in response to increasing fertilizer addition in maize (Zea mays L.) agriculture in western Kenya," Nutrient Cycling in Agroecosystems, vol. 100, no. 2, pp. 177-187, 2014.

[41] J. E. Hickman, K. L. Tully, P. M. Groffman, W. Diru, and C. A. Palm, "A potential tipping point in tropical agriculture: avoiding rapid increases in nitrous oxide fluxes from agricultural intensification in Kenya," Journal of Geophysical Research: Biogeosciences, vol. 120, no. 5, pp. 938-951, 2015.

[42] T. S. Rosenstock, M. Mpanda, D. E. Pelster et al., "Greenhouse gas fluxes from agricultural soils of Kenya and Tanzania," Journal of Geophysical Research: Biogeosciences, vol. 121, no. 6, pp. 1568-1580, 2016.

[43] D. Ortiz-Gonzalo, A. de Neergaard ., P. Vaast, V. SuárezVillanueva, M. Oelofse, and T. S. Rosenstock, "Multi-scale measurements show limited soil greenhouse gas emissions in Kenyan smallholder coffee-dairy systems," The Science of the Total Environment, vol. 626, pp. 328-339, 2018.

[44] I. Wanyama, D. E. Pelster, C. Arias-Navarro, K. ButterbachBahl, L. V. Verchot, and M. C. Rufino, "Management intensity controls soil $\mathrm{N}_{2} \mathrm{O}$ fluxes in an Afromontane ecosystem," The Science of the Total Environment, vol. 624, pp. 769-780, 2018.

[45] W. K. Atakora, P. K. Kwakye, D. Weymann, and N. Brüggemann, "Stimulus of nitrogen fertilizers and soil characteristics on maize yield and nitrous oxide emission from Ferric Luvisol in the Guinea Savanna agro-ecological zone of Ghana," Scientific African, vol. 6, Article ID e00141, 2019.

[46] J. Zheng, Y. Qu, M. M. Kilasara, W. N. Mmari, and S. Funakawa, "Soil-atmosphere exchange of nitrous oxide in two Tanzanian croplands: effects of nitrogen and stover management," Agricultural and Forest Meteorology, vol. 275, pp. 24-36, 2019.

[47] S. G. Raji and P. Dörsch, "Effect of legume intercropping on $\mathrm{N}_{2} \mathrm{O}$ emissions and $\mathrm{CH}_{4}$ uptake during maize production in the Great Rift Valley, Ethiopia," Biogeosciences, vol. 17, no. 2, pp. 345-359, 2020.

[48] C. M. Musafiri, J. M. Macharia, M. N. Kiboi et al., "Soil greenhouse gas fluxes from maize cropping system under different soil fertility management technologies in Kenya," Agriculture, Ecosystems \& Environment, vol. 301, Article ID 107064, 2020.

[49] R. Chikowo, P. Mapfumo, P. Nyamugafata, and K. E. Giller, "Maize productivity and mineral $\mathrm{N}$ dynamics following different soil fertility management practices on a depleted sandy soil in Zimbabwe," Agriculture, Ecosystems \& Environment, vol. 102, no. 2, pp. 119-131, 2004.

[50] N. Millar, J. K. Ndufa, G. Cadisch, and L. Baggs, "Nitrous oxide emissions following incorporation of improved-fallow residues in the humid tropics," Global Biogeochemical Cycles, vol. 18, pp. 1-9, 2004.

[51] L. Chapuis-Lardy, A. Metay, M. Martinet et al., "Nitrous oxide fluxes from Malagasy agricultural soils," Geoderma, vol. 148, no. 3-4, pp. 421-427, 2009.

[52] F. Mapanda, M. Wuta, J. Nyamangara, and R. M. Rees, "Effects of organic and mineral fertilizer nitrogen on greenhouse gas emissions and plant-captured carbon under maize cropping in Zimbabwe," Plant and Soil, vol. 343, no. 12, pp. 67-81, 2011.

[53] G. Nyamadzawo, Y. Shi, N. Chirinda et al., "Combining organic and inorganic nitrogen fertilisation reduces $\mathrm{N}_{2} \mathrm{O}$ emissions from cereal crops: a comparative analysis of China and Zimbabwe," Mitigation and Adaptation Strategies for Global Change, vol. 22, no. 2, pp. 233-245, 2017.

[54] B. K. Kurgat, S. Stöber, S. Mwonga, H. Lotze-Campen, and T. S. Rosenstock, "Livelihood and climate trade-offs in Kenyan peri-urban vegetable production," Agricultural Systems, vol. 160, pp. 79-86, 2018.

[55] K. Roing, A. Goosens, and J. Diels, "Initial nitrous oxide fluxes from a maize-legume cropping system in a soil of the derived Savanna zone of Nigeria-effect of fertilizer and incorporated organic matter," West African Journal of Applied Ecology, vol. 6, pp. 47-54, 2004.

[56] J. M. Kimetu, D. N. Mugendi, A. Bationo et al., "Partial balance of nitrogen in a maize cropping system in humic nitisol of Central Kenya," Nutrient Cycling in Agroecosystems, vol. 76, pp. 521-530, 2007.

[57] J. Masaka, J. Nyamangara, and M. Wuta, "Nitrous oxide emissions from wetland soil amended with inorganic and organic fertilizers," Archives of Agronomy and Soil Science, vol. 60, no. 10, pp. 1363-1387, 2014.

[58] N. Fatumah, L. K. Munishi, and P. A. Ndakidemi, "Variations in greenhouse gas fluxes in response to short-term changes in weather variables at three elevation ranges, wakiso district, Uganda," Atmosphere, vol. 10, no. 11, p. 708, 2019.

[59] A. Olaleye, D. Peak, A. Shorunke et al., "Effect of manure and urea fertilization on yield, carbon speciation and greenhouse gas emissions from vegetable production systems of Nigeria and republic of Benin: a phytotron study," Agronomy, vol. 10, pp. 1-20, 2020.

[60] C. A. O. Midega, J. O. Pittchar, J. A. Pickett, G. W. Hailu, and Z. R. Khan, "A climate-adapted push-pull system effectively controls fall armyworm, Spodoptera frugiperda (J E Smith), in maize in East Africa," Crop Protection, vol. 105, pp. 10-15, 2018.

[61] M. Zhou, P. Brandt, D. Pelster, M. C. Rufino, T. Robinson, and K. Butterbach-Bahl, "Regional nitrogen budget of the lake Victoria Basin, East Africa: syntheses, uncertainties and perspectives," Environmental Research Letters, vol. 9, no. 10, Article ID 105009, 2014.

[62] J. Mugwe, F. Ngetich, and E. O. Otieno, Integrated Soil Fertility Management in Sub-Saharan Africa: Evolving Paradigms toward Integration, Springer, Cham, Switzerland, 2019.

[63] J. M. Melillo, J. D. Aber, and J. F. Muratore, "Nitrogen and lignin control of hardwood leaf litter decomposition dynamics," Ecology, vol. 63, no. 3, pp. 621-626, 1982.

[64] J. Dick, U. Skiba, R. Munro, and D. Deans, "Effect of N-fixing and non $\mathrm{N}$-fixing trees and crops on $\mathrm{NO}$ and $\mathrm{N}_{2} \mathrm{O}$ emissions from Senegalese soils," Journal of Biogeography, vol. 33, no. 3, pp. 416-423, 2006.

[65] A. Gütlein, F. Gerschlauer, I. Kikoti, and R. Kiese, "Impacts of climate and land use on $\mathrm{N}_{2} \mathrm{O}$ and $\mathrm{CH}_{4}$ fluxes from tropical ecosystems in the Mt. Kilimanjaro region, Tanzania," Global Change Biology, vol. 24, no. 3, pp. 1239-1255, 2018.

[66] B. Vanlauwe, K. Descheemaeker, K. E. Giller et al., "Integrated soil fertility management in Sub-Saharan Africa: unravelling local adaptation," Soils, vol. 1, no. 1, pp. 491-508, 2015.

[67] J. Macharia, J. Mugwe, M. Mucheru-Muna, and D. Mugendi, "Socioeconomic factors influencing levels of knowledge in soil fertility management in the central highlands of Kenya," Journal of Agricultural Science and Technology A, vol. 4, pp. 701-711, 2014.

[68] W. B. Nganga, K. O. Ng'etich, M. J. Macharia, N. M. Kiboi, N. Adamtey, and K. F. Ngetich, "Multi-influencing-factors' evaluation for organic-based soil fertility technologies out- 
scaling in Upper Tana Catchment in Kenya," Scientific African, vol. 7, Article ID e00231, 2020.

[69] M. N. Kiboi, K. F. Ngetich, D. N. Mugendi, A. Muriuki, N. Adamtey, and A. Fliessbach, "Microbial biomass and acid phosphomonoesterase activity in soils of the Central Highlands of Kenya," Geoderma Regional, vol. 15, Article ID e00193, 2018.

[70] J. Masaka, J. Nyamangara, and M. Wuta, "Nitrous oxide emission from wetland soil following single and seasonal split application of cattle manure to field tomato (Lycopersicon esculentum, Mill var. Heinz) and rape (Brassica napus, L. var. Giant) crops," SpringerPlus, vol. 5, 2016.

[71] R. Sommer, J. Mukalama, J. Kihara, S. Koala, L. Winowiecki, and D. Bossio, "Nitrogen dynamics and nitrous oxide emissions in a long-term trial on integrated soil fertility management in Western Kenya," Nutrient Cycling in Agroecosystems, vol. 105, no. 3, pp. 229-248, 2016.

[72] J. W. Van Groenigen, G. L. Velthof, O. Oenema, K. J. Van Groenigen, and C. Van Kessel, "Towards an agronomic assessment of $\mathrm{N}_{2} \mathrm{O}$ emissions: a case study for arable crops," European Journal of Soil Science, vol. 61, no. 6, pp. 903-913, 2010.

[73] K. A. Frimpong, D. O. Yawson, E. M. Baggs, and K. Agyarko, "Does incorporation of cowpea-maize residue mixes influence nitrous oxide emission and mineral nitrogen release in a tropical luvisol?" Nutrient Cycling in Agroecosystems, vol. 91, no. 3, pp. 281-292, 2011.

[74] C. Oertel, J. Matschullat, K. Zurba, F. Zimmermann, and S. Erasmi, "Greenhouse gas emissions from soils-a review," Geochemistry, vol. 76, no. 3, pp. 327-352, 2016.

[75] H. F. Birch, "The effect of soil drying on humus decomposition and nitrogen availability," Plant and Soil, vol. 10, no. 1, pp. 9-31, 1958.

[76] M. Rabenarivo, N. Wrage-Moennig, J.-L. Chotte, L. Rabeharisoa, T. M. Razafimbelo, and L. Chapuis-Lardy, "Emissions of $\mathrm{CO}_{2}$ and $\mathrm{N}_{2} \mathrm{O}$ from a pasture soil from Madagascar-simulating conversion to direct-seeding mulchbased cropping in incubations with organic and inorganic inputs," Journal of Plant Nutrition and Soil Science, vol. 177, no. 3, pp. 360-368, 2014.

[77] M. H. Chantigny, P. Rochette, D. A. Angers et al., "Soil nitrous oxide emissions following band-incorporation of fertilizer nitrogen and swine manure," Journal of Environmental Quality, vol. 39, no. 5, pp. 1545-1553, 2010.

[78] Y. Zhu, L. Merbold, S. Leitner et al., "Influence of soil properties on $\mathrm{N}_{2} \mathrm{O}$ and $\mathrm{CO}_{2}$ emissions from excreta deposited on tropical pastures in Kenya," Soil Biology and Biochemistry, vol. 140, Article ID 107636, 2020.

[79] D. E. Pelster, M. H. Chantigny, P. Rochette, D. A. Angers, C. Rieux, and A. Vanasse, "Nitrous oxide emissions respond differently to mineral and organic nitrogen sources in contrasting soil types," Journal of Environmental Quality, vol. 41, no. 2, pp. 427-435, 2012.

[80] L. R. Bakken, L. Bergaust, B. Liu, and A. Frostegård, "Regulation of denitrification at the cellular level: a clue to the understanding of $\mathrm{N}_{2} \mathrm{O}$ emissions from soils," Philosophical Transactions of the Royal Society B: Biological Sciences, vol. 367, no. 1593, pp. 1226-1234, 2012.

[81] L. Chapuis-Lardy, N. Wrage, A. Metay, J.-L. Chotte, and M. Bernoux, "Soils, a sink for $\mathrm{N}_{2} \mathrm{O}$ ? A review," Global Change Biology, vol. 13, pp. 1-17, 2007.

[82] J. Barron and G. Okwach, "Run-off water harvesting for dry spell mitigation in maize (Zea mays L.): results from on-farm research in semi-arid Kenya," Agricultural Water Management, vol. 74, no. 1, pp. 1-21, 2005.

[83] B. Vanlauwe and K. Giller, "Popular myths around soil fertility management in sub-Saharan Africa," Agriculture, Ecosystems \& Environment, vol. 116, no. 1-2, pp. 34-46, 2006.

[84] M. Tongwane, T. Mdlambuzi, M. Moeletsi, M. Tsubo, V. Mliswa, and L. Grootboom, "Greenhouse gas emissions from different crop production and management practices in South Africa," Environmental Development, vol. 19, pp. 23-35, 2016.

[85] M. Fosu, R. F. Kuhne, and P. L. G. Vlek, "Mineralization and microbial biomass dynamics during decomposition of four leguminous residues," Journal of Biological Sciences, vol. 7, no. 4, pp. 632-637, 2007.

[86] N. Z. Lupwayi, A. C. Kennedy, and R. M. Chirwa, "Grain legume impacts on soil biological processes in sub-Saharan Africa," African Journal of Plant Science, vol. 5, pp. 1-7, 2011.

[87] M. Bekunda, N. Sanginga, and P. L. Woomer, "Restoring soil fertility in sub-Sahara Africa," Advances in Agronomy, vol. 108, pp. 183-236, 2010.

[88] R. Sommer, D. Bossio, L. Desta et al., Profitable and Sustainable Nutrient Management Systems for East and Southern African Smallholder Farming Systems Challenges and Opportunities: A Synthesis of the Eastern and Southern Africa Situation in Terms of Past Experiences, Present and Future Opportunities in Promoting Nutrients Use in Africa, The University of Queensland, Brisbane, Australia, 2013.

[89] B. C. G. Kamanga, S. R. Waddington, M. J. Robertson, and K. E. Giller, "Risk analysis of maize-legume crop combinations with smallholder farmers varying in resource endowment in central Malawi," Experimental Agriculture, vol. 46, no. 1, pp. 1-21, 2010.

[90] E. Nafi, H. Webber, I. Danso, J. B. Naab, M. Frei, and T. Gaiser, "Interactive effects of conservation tillage, residue management, and nitrogen fertilizer application on soil properties under maize-cotton rotation system on highly weathered soils of West Africa," Soil and Tillage Research, vol. 196, Article ID 104473, 2020. 